\title{
Bioethanol Production from Sugarcane Press-Mud: Assessment of the Fermentation Conditions to Reduce Fusel Alcohol
}

\author{
Nestor Sanchez ${ }^{1}$, Martha Cobo ${ }^{1}$, David Rodriguez-Fontalvo ${ }^{1}$, Miguel Ángel Uribe-Laverde ${ }^{2}$ \\ and Ruth Y. Ruiz-Pardo ${ }^{3, *}$
}

1 Energy, Materials, and Environmental Laboratory, Department of Chemical Engineering and Biochemical Processes, Faculty of Engineering, Campus Universitario Puente del Común, Universidad de La Sabana, Km. 7 Autopista Norte, Bogotá 140013, Colombia; nestor.sanchez1@unisabana.edu.co (N.S.); martha.cobo@unisabana.edu.co (M.C.); Davidrodfo@unisabana.edu.co (D.R.-F.)

2 Grupo de Física y Matemáticas Aplicadas, Faculty of Engineering, Campus Universitario Puente del Común, Universidad de La Sabana, Km. 7 Autopista Norte, Bogotá 140013, Colombia; miguel.uribe1@unisabana.edu.co

3 Grupo de Investigación en Procesos Agroindustriales, Faculty of Engineering, Campus Universitario Puente del Común, Universidad de La Sabana, Km. 7 Autopista Norte, Bogotá 140013, Colombia

* Correspondence: ruth.ruiz@unisabana.edu.co; Tel.: +57-1-861-5555 (ext. 25011)

\section{check for}

updates

Citation: Sanchez, N.; Cobo, M.; Rodriguez-Fontalvo, D.; Uribe-

Laverde, M.Á.; Ruiz-Pardo, R.Y.

Bioethanol Production from

Sugarcane Press-Mud: Assessment of the Fermentation Conditions to Reduce Fusel Alcohol. Fermentation 2021, 7, 194. https://doi.org/ 10.3390/fermentation7030194

Academic Editor: Luísa

Seuanes Serafim

Received: 3 August 2021

Accepted: 10 September 2021

Published: 15 September 2021

Publisher's Note: MDPI stays neutral with regard to jurisdictional claims in published maps and institutional affiliations.

Copyright: (c) 2021 by the authors. Licensee MDPI, Basel, Switzerland. This article is an open access article distributed under the terms and conditions of the Creative Commons Attribution (CC BY) license (https:// creativecommons.org/licenses/by/ $4.0 /)$.
Abstract: Within a biorefinery context, bioethanol is a promising platform molecule since it can be used as raw material to produce a wide spectrum of valuable industrial products such as $\mathrm{H}_{2}$ and light olefins. However, the presence of impurities limits the conversion of bioethanol in these products. Herein, we aimed to determine the proper pretreatment and fermentation conditions to yield bioethanol with a low content of impurities, such as 3-methyl-1-butanol, by using sugarcane press-mud as feedstock. To do so, a Box-Behnken methodology was employed to select proper pretreatment and fermentation conditions. Factors assessed were temperature, stirring, and $\mathrm{pH}$ during fermentation of hydrolysates coming from two different pretreatment methods named as hydrothermal and acid hydrolysis. Results showed that the fermentation temperature should be kept between $26-30{ }^{\circ} \mathrm{C}$ to assure at least $91 \mathrm{~g} / \mathrm{L}$ ethanol. The fusel alcohol content would be reduced by $22 \%$ at $30{ }^{\circ} \mathrm{C}, \mathrm{pH}=4.5$, and $200 \mathrm{rpm}$ if sugarcane press-mud is pretreated under acid hydrolysis conditions $\left(\mathrm{T}=130{ }^{\circ} \mathrm{C}, \mathrm{t}=1 \mathrm{~h}, 16 \mathrm{~g} \mathrm{HNO}_{3} / \mathrm{kg}\right.$ solid). Further studies should aim to integrate these conditions within a biorefinery concept to yield valuable products such as $\mathrm{H}_{2}$ and ethylene.

Keywords: 3-methyl-1-butanol; acid hydrolysis; Box-Behnken design; inhibitors; yeast strains

\section{Introduction}

Decarbonizing the economy by using biomass and biofuels is necessary to meet the agenda stated by the United Nations (UN) associated with sustainable development goals (SDG) [1]. Bioethanol is an interested feedstock since it can be used as a building block to produce a wide variety of important products, making it attractive for shifting from traditional oil refineries into biorefineries [2]. These products include hydrogen $\left(\mathrm{H}_{2}\right)$, light olefins, gasoline, and acetonitrile [3-5]. Bioethanol can be produced from a wide variety of biomass feedstocks including sugarcane, cornstarch, sugar beet, wheat, rye, lignocellulosic material, potato, cassava, sugarcane press-mud, and pineapple industrial wastes [3,6-8]. However, the complex composition of bioethanol affects the production of $\mathrm{H}_{2}$ and light olefins. For instance, bioethanol conversion to $\mathrm{H}_{2}$ by ethanol steam reforming (ESR) is strongly influenced by the presence of impurities such as fusel alcohols, sulfur components, and acetic acid [3]. Besides, the presence of those impurities affects the conversion of ethanol into light olefins through dehydration reactions [9]. Since impurities have a detrimental effect on bioethanol upgrade, it is pertinent to seek alternatives to mitigate their formation. Those alternatives might include controlling the formation of impurities in upstream processes such as pretreatment, fermentation, and/or distillation, 
and designing resistant catalysts for upgrading processes to overcome the negative impacts of impurities. While the last strategy has been widely discussed in the literature [3], there are few reports concerning the first one. For instance, Sanchez et al. [10] assessed the effect of supplementation on fermentation to produce bioethanol for its further conversion to $\mathrm{H}_{2}$ by ESR over $\mathrm{RhPt} / \mathrm{CeO}_{2}-\mathrm{SiO}_{2}$ at $700{ }^{\circ} \mathrm{C}$, concluding that 3-methyl-1-butanol had an important effect during ESR ascribed to the formation of carbon deposits. Rossetti et al. [11] evaluated different purification alternatives to produce bioethanol for its subsequent conversion to ethylene. They showed that it is possible to mitigate the effect of impurities if a by-pass stream is employed along with the distillation unit. Recently, we reported an alternative to mitigate the production of fusel alcohol during the pretreatment of sugarcane press-mud and subsequent fermentation with aims to yield $\mathrm{H}_{2}$ by ESR. This alternative requires the use of $\mathrm{HNO}_{3}$ at $130{ }^{\circ} \mathrm{C}$ for $1 \mathrm{~h}$ to increase the $\mathrm{H}_{2}$ yield by at least $20 \%$ [7]. The integration of alternatives in the upstream processes is key to mitigate the formation of bioethanol impurities and enhance the conversion of bioethanol into valuable products by catalytic pathways.

Among bioethanol impurities, fusel alcohols and acetic acid are considered as the main components yielded during fermentation. Glycerol is also produced in high quantities, but due to its high boiling point it is easily removed by simple distillation [3,10]. Fusel alcohol, such as 1-propanol, 2-methyl-1-propanol, and 3-methyl-1-butanol, are formed during fermentation by either of the following pathways: (i) Ehrlich metabolic pathway or (ii) novo synthesis pathway [12]. Their formation is influenced by different variables such as nitrogen, temperature, $\mathrm{pH}$, dissolved oxygen, and yeast strain $[12,13]$. For instance, we reported that the addition of ammonium sulfate $\left(\left(\mathrm{NH}_{4}\right)_{2} \mathrm{SO}_{4}\right)$ decreased the amount of fusel alcohol, mainly 2-methyl-1-propanol, during the fermentation of sugarcane press-mud at $30{ }^{\circ} \mathrm{C}$ [14]. Arshad et al. [15] assessed the effect of several fermentation variables, such as substrate concentration, $\mathrm{pH}$, and inoculum size, over the fusel alcohol concentration during fermentation of sugarcane molasses at $30{ }^{\circ} \mathrm{C}$. They concluded that all the assessed variables have a significant effect $(p<0.05)$ over the formation of fusel alcohols. Similarly, Rollero et al. [16] reported that nitrogen content and temperature had an important effect on the production of 2-methyl-1-propanol during the fermentation of synthetic grape culture medium. Saerens et al. [17] described that the main effect of temperature, during the production of fusel alcohol, was ascribed to the gene expression that encodes the enzymes associated with the production of fusel alcohol during fermentation. Aside from the aforementioned variables, the biomass source affects the production of fusel alcohols [18]. Similarly, pretreatment conditions of biomass have an important effect on the fermentation of hydrolysates in Jerusalem stalks [19] and sugarcane press-mud [7], since pretreatment will affect the composition of reducing sugars [20]. Also, yeast strains have an influence on the bioethanol profile and ethanol productivity [21-23]. Therefore, it is important to evaluate different yeast strains to mitigate the fusel alcohol content without significantly affecting the ethanol production.

Sugarcane press-mud is an agro-industrial waste obtained in non-centrifugal sugar facilities in countries such as India and Colombia [10]. Bioethanol production from this waste has been recently reported from a technical point of view. However, the presence of fusel alcohols will limit its conversion into valuable products such as $\mathrm{H}_{2}$ and ethylene. Recently, we reported that upgrading sugarcane press-mud into $\mathrm{H}_{2}$ is feasible if fusel alcohols are reduced by $20 \%$ [7]. To date, only the effect of pretreatment conditions on fusel alcohol has been explored. However, as previously described, fermentation factors such as yeast strains, temperature, $\mathrm{pH}$, and stirring will have an impact on bioethanol production. Therefore, the aim of this paper is to determine the effect of the aforecited variables on the ethanol and fusel alcohol content. To do so, an initial screening on the yeast strain was explored. Herein, three industrial yeast strains, named as HG-1, M-1, and C-70, were used. Those strains are supplied by Fermentis, and all can be used for very high gravity fermentation. Strain HG-1, hereafter named as Ethanol Red, has been employed for producing bioethanol from sugarcane press-mud [10,14,24]. In addition, it has been 
used for producing beverage spirits like vodka, where the optimum operating temperature ranges between 25 and $33^{\circ} \mathrm{C}$. Strain C-70 is employed due to its high attenuation and ethanol tolerance. Besides, it is used for low gravity fermentation with a wide variety of feedstocks such as sugarcane juice and molasses for producing rum [25]. This strain could be employed for fermentation carried out between 25 and $33^{\circ} \mathrm{C}$. Lastly, strain M-1 is highly employed for producing scotch whisky, whose optimum temperature ranges between 20 and $31{ }^{\circ} \mathrm{C}$ [26]. Afterwards, fermentation conditions were selected by using the response surface methodology to seek possible interaction among factors tested (i.e., temperature, $\mathrm{pH}$, and stirring). This study gives insights on the alternatives to produce bioethanol while reducing the content of fusel alcohol for further integration within a biorefinery.

\section{Materials and Methods}

\subsection{Material Collection}

The sugarcane press-mud employed in this study was collected from the Andean region in Colombia with the following average composition: $69.79 \pm 5.98 \%$ moisture; $22.95 \pm 4.62 \%$ carbohydrates; $3.19 \pm 1.58 \%$ wax; $1.12 \pm 1.10 \%$ crude fiber; $2.08 \pm 0.53$ ashes; $0.87 \pm 0.56 \%$ proteins; and $\mathrm{pH}$ of $5.75 \pm 0.93$ [10]. Another sample, collected from the municipality of Villeta $\left(5^{\circ} 00^{\prime} 46^{\prime \prime} \mathrm{N} ; 74^{\circ} 28^{\prime} 23^{\prime \prime} \mathrm{W}\right)$ with a composition of $76.20 \%$ moisture, $18.33 \%$ carbohydrates, $2.17 \%$ proteins, $1.70 \%$ fat, and $1.60 \%$ ashes, was employed to validate the experiments. This sample is named Villeta throughout the manuscript. Samples were transported to Universidad de La Sabana (Bogotá, Colombia), stored in $50 \mathrm{~mL}$ flasks, and kept at $0{ }^{\circ} \mathrm{C}$.

\subsection{Evaluation of Yeast Strains}

A one-factor experimental design method at three different levels was employed to determine the suitable yeast strains. Experiments were performed by three independent replicates. HG-1, M-1, and C-70 yeast strains (Fermentis by Lesaffre, Marcq-en-Baroeul, France) were evaluated in terms of ethanol and fusel alcohol concentration after the fermentation of hydrolysates coming from sugarcane press-mud pretreatment. To do so, $0.53 \pm 0.04 \mathrm{~g} / \mathrm{L}$ of dry yeast was inoculated in YPD (Yeast extract $10 \mathrm{~g} / \mathrm{L}$, peptone $20 \mathrm{~g} / \mathrm{L}$, and glucose $20 \mathrm{~g} / \mathrm{L}$ ) at $30^{\circ} \mathrm{C}$ and $200 \mathrm{rpm}$ until the concentration was about $1 \times 10^{7}$ colony forming unit per $\mathrm{mL}(\mathrm{CFU} / \mathrm{mL})$. Fermentation of sugarcane press-mud hydrolysates by using those yeast strains was performed as described in Section 2.4. Quantification of yeast cell viability, carbohydrates conversion, ethanol, and fusel alcohol concentration was done according to procedures described in Section 2.6. Data were analyzed with SPSS software V26 (IBM, Armonk, NY, USA). A Duncan test $(p<0.05)$ was performed to determine significant differences among the yeast strains.

\subsection{Sample Preparation}

Sugarcane press-mud samples collected from the Andean region had a total solid content of $30 \mathrm{wt} . \%$ according to the characterization described in Section 2.1. Total solid content was determined by the oven method described by the National Renewable Energy Laboratory (NREL), who report that total solids represent the amount of solids remaining after drying the sample at $105^{\circ} \mathrm{C}$. [27]. To assess the effect of the fermentation conditions, sugarcane press-mud samples were diluted with water until a solid concentration of $29 \mathrm{wt} . \%$ was obtained. Under this condition, a high ethanol content was assured [10]. Then, samples were hydrolyzed in an autoclave (Tomy SX 700, Tomy Digital-Biology Co., LTD., Tokyo, Japan) at $130^{\circ} \mathrm{C}$ for $1 \mathrm{~h}$. Samples were hydrolyzed under two different hydrolysis conditions, the former without $\mathrm{HNO}_{3}$, hereafter named as hydrothermal, and the latter with an addition of $16 \mathrm{~g}$ of $\mathrm{HNO}_{3}$ per dry solid, hereafter named as acid hydrolysis. The reaction was stopped by placing the sample in an ice water bath. After that, sugarcane press-mud was sieved through a 70-mesh sieve $(212 \mu \mathrm{m})$. The recovered liquid was supplemented with peptone, yeast extract, $\mathrm{MgSO}_{4} \cdot 7 \mathrm{H}_{2} \mathrm{O}, \mathrm{Ca}_{3}\left(\mathrm{PO}_{4}\right)_{2}$, and $\left(\mathrm{NH}_{4}\right)_{2} \mathrm{SO}_{4}$ [10]. The supplemented culture medium was split into 3 similar fractions for each hydrolysis 
condition. The $\mathrm{pH}$ of the samples was adjusted to $4.5,5.5$, and 6.5 by using either $3 \mathrm{M}$ $\mathrm{HNO}_{3}$ or $3 \mathrm{M} \mathrm{NaOH}$ aqueous solutions. All samples were sterilized in an autoclave (Tomy SX 700, Tomy Digital-Biology Co., LTD., Tokyo, Japan) at $121^{\circ} \mathrm{C}$ for $15 \mathrm{~min}$.

\subsection{Fermentation}

Samples were fermented in $15 \mathrm{~mL}$ centrifuge tubes with a total volume of $4 \mathrm{~mL}$ and an inoculum size of $10 \mathrm{vol} \%$. Inoculum was prepared according to previous studies [14]. Dry Saccharomyces cerevisiae (Ethanol Red ${ }^{\circledR}$, Fermentis by Lesaffre, Marcq-en-Baroeul, France) was activated in water at $30{ }^{\circ} \mathrm{C}$ for $20 \mathrm{~min}$. After that, yeast was cultivated in Potato Dextrose Agar (PDA) (Oxoid, New Hampshire, England) at $30^{\circ} \mathrm{C}$ during $48 \mathrm{~h}$ and propagated in YPD culture (i.e., glucose $20 \mathrm{~g} / \mathrm{L}$, peptone $20 \mathrm{~g} / \mathrm{L}$, yeast extract $10 \mathrm{~g} / \mathrm{L}$ ) until yeast concentration reached about $10^{7} \mathrm{CFU} / \mathrm{mL}$. Fermentation was performed in a shaker (Innova 42, Eppendorf, Hamburg, Germany), at the stirring and fermentation conditions described in Section 2.5, for $24 \mathrm{~h}$. After fermentation time, samples were characterized in terms of yeast cell viability, carbohydrates conversion, ethanol, 1-propanol, 2-methyl1-propanol, 3-methyl-1-butanol, and acetic acid concentration according to the methods described in Section 2.7.

\subsection{Experimental Design}

A Box-Behnken design method (BBD) was employed to determine the effects of selected fermentation factors such as $\mathrm{pH}(\mathrm{A})$, stirring (B), temperature (C), and hydrolysis (D). This method allows for defining whether the factors or their interaction have an effect over the response variables with less experimental units, compared to a factorial experiment [28]. Walker and Stewart [29] prompt that yeast such as $S$. cerevisiae grows well between 20 and $30^{\circ} \mathrm{C}$ and $\mathrm{pH} 4.5$ and 6.5. Besides, a stirring rate up to $200 \mathrm{rpm}$ is advisable to produce ethanol by fermentation [30]. Hence, each numerical factor, such as $\mathrm{pH}$, stirring, and temperature, was set to 3 levels according to the above parameters: $\mathrm{pH}\left(4.5,5.5\right.$, and 6.5); stirring $(0 \mathrm{rpm}, 100 \mathrm{rpm}$, and $200 \mathrm{rpm})$; and temperature $\left(20^{\circ} \mathrm{C}\right.$, $25^{\circ} \mathrm{C}$, and $\left.30^{\circ} \mathrm{C}\right)$. Those values agree with the optimal growth temperature $\left(25-35^{\circ} \mathrm{C}\right)$ suggested by the supplier. Otherwise, hydrolysis conditions were set as categorical factors to 2 levels, named as hydrothermal and acid hydrolysis, according to previous results [7]. Therefore, 34 independent experiments were carried out. Experimental data was adjusted to a second-order polynomial model as shown in Equation (1), where $\mathrm{Y}$ represents the response variable (i.e., yeast cell viability, carbohydrates conversion, ethanol content, and fusel alcohol content) and $\beta_{\mathrm{i}}, \beta_{\mathrm{ii}}$, and $\beta_{\mathrm{ij}}$ represent the regression coefficients for intercept, linearity, and quadratic terms, respectively. In addition, $\mathrm{i}$ and $\mathrm{j}$ represent the factors $\mathrm{A}, \mathrm{B}$, and C [31].

$Y=\beta_{0}+\beta_{A} A+\beta_{B} B+\beta_{C} C+\beta_{A B} A B+\beta_{A C} A C+\beta_{B C} B C+\beta_{A A} A^{2}+\beta_{B B} B^{2}+\beta_{C C} C^{2}$.

The statistical analysis was performed in the software design expert, version V.7.1.0 (Stat-Ease Inc., Minneapolis, MN, USA). Besides, the significance of the model was determined through an analysis of variance (ANOVA) and the fit model was assessed by the correlation coefficient $\left(R^{2}\right)$ and the lack of fit values.

\subsection{Validation Results}

Villeta samples were employed as feedstock to validate the experimental conditions found previously. Samples were pretreated and fermented under the same procedure described in Sections 2.2 and 2.3. Validation was performed in terms of yeast cell viability, carbohydrates conversion, ethanol, and fusel alcohol content after fermentation at the selected conditions after the pretreatment stage.

\subsection{Analytical Methods}

Yeast cell viability was quantified through the counting plate agar method [32]. Yeast cells were grown on PDA after $48 \mathrm{~h}$ incubation. Yeast cell viability, expressed as log units of 
colony-forming units per $\mathrm{mL}(\log \mathrm{CFU} / \mathrm{mL})$, was calculated before and after fermentation. Differences between both states were reported. Carbohydrates were quantified spectrophotometrically by the phenol-sulfuric method by using microwells [10]. Absorbance was read at $490 \mathrm{~nm}$ in a microwell absorbance reader (iMarkTM, Bio-Rad, Hercules, CA, USA). The amount of carbohydrates was estimated by employing glucose (Carlos Erba, Cornaredo, Italy) as standard (0-500 $\mu \mathrm{g} / \mathrm{mL})$ and expressed as glucose equivalent per liter (GE/L). Carbohydrate conversion was calculated according to Equation (2), where $X_{c}$ is the carbohydrate conversion, $\mathrm{G}_{0}$ is the initial carbohydrate content, and $\mathrm{Gf}$ is the carbohydrate content after fermentation.

$$
\mathrm{X}_{\mathrm{C}}=\frac{\mathrm{G}_{0}-\mathrm{G}_{\mathrm{F}}}{\mathrm{G}_{0}}
$$

Ethanol, 1-propanol, 2-methyl-1-propanol, 3-methyl-1-butanol, acetic acid, and HMF were quantified using a Clarus 580 GC (GC, Perkin Elmer, Waltham, MA, USA), equipped with an Elite wax ETR column (60 m, 0.25 mm ID, Perkin Elmer, Waltham, MA, USA) connected to a Flame Ionization Detector (FID). Both detector and injector temperatures were adjusted to $240{ }^{\circ} \mathrm{C}$ [10].

This study aimed to identify the effect of hydrolysis conditions and fermentation parameters, such as $\mathrm{pH}$, temperature, and stirring, on ethanol and fusel alcohol. To do so, an initial screen of the yeast was done following by a Box-Behnken design method to determine the effect of the above variables. The Box-Behnken design method was employed to minimize the number of experimental runs and to identify possible interactions among the tested factors and their influence, mainly on the production of ethanol and fusel alcohol content.

\section{Result}

\subsection{Yeast Screening}

The strains used in a fermentation can yield different distributions of fermentation products depending on the application [21,22]. Herein, it is important to employ a yeast strain that will achieve the higher amount of ethanol while lessening the fusel alcohol content. In addition, larger sugar consumption is a desirable characteristics during ethanol production [33]. Table 1 shows the performance of three different yeast strains (i.e., HG-1, $\mathrm{M}-1$, and C-70) in terms of yeast cell viability, carbohydrate conversion, ethanol, and fusel alcohol concentration when sugarcane press-mud was hydrolyzed and fermented according to previous experiments [7]. Yeast cell viability is an outstanding parameter for designing industrial processes [32]. According to Table 1, the M-1 strain showed the highest yeast cell viability $(2.03 \pm 0.01 \mathrm{Log} C F U / \mathrm{mL})$, followed by the HG-1 strain $(1.89 \pm 0.05 \mathrm{Log} \mathrm{CFU} / \mathrm{mL})$ and C-70 strain $(1.69 \pm 0.12 \mathrm{Log}$ CFU/mL), which implies that the M-1 strain adapts better to the sugarcane press-mud fermentation culture than the other strains. Concerning carbohydrate conversion, all strains showed a similar behavior and consequently, the ethanol concentration was similar among strains according to the ANOVA test $(p<0.05)$, as shown in Table 1. Similar results were observed by Kawa-Rygielska et al. [34], who reported an ethanol concentration of $92 \mathrm{~g} / \mathrm{L}$ after $72 \mathrm{~h}$ fermentation of sugar beet juice at a temperature of $30{ }^{\circ} \mathrm{C}, \mathrm{pH}$ of 5.0 , and $150 \mathrm{rpm}$ with C-70 and HG-1 strains. Likewise, Askarbekov et al. [35] reported an ethanol concentration of $12 \mathrm{vol} . \%$ after the fermentation of sweet sorghum juice at $26^{\circ} \mathrm{C}$ with $\mathrm{C}-70, \mathrm{HG}-1$, and $\mathrm{M}-1$ yeast strains. Therefore, those strains produce similar ethanol concentration. Concerning the fusel alcohol concentration, 3-methyl-1-butanol concentration was significantly different among strains according to Duncan test $(p<0.05)$. For instance, HG-1 produced less of this alcohol $(176.01 \mathrm{mg} / \mathrm{L})$, followed by C-70 (228.69 mg/L) and M-1 (244.94 mg/L). Askarbekov et al. [35] also compared the production of those yeast strains during the fermentation of sweet sorghum juice at $26{ }^{\circ} \mathrm{C}$. They reported that M-1 produces less of 3-methyl-1-butanol $(183 \mathrm{mg} / \mathrm{L})$, followed by HG-1 (288 mg/L) and C-70 (338 mg/L). Differences between both studies show that fermentation conditions and feedstock affect the production of 3-methyl-1-butanol when yeast strains such as C-70, HG-1, and M-1 are employed. Those streams were 
commonly employed for producing beverage spirits such as rum, vodka, and whisky [26]. Therefore, it is expected that the ethanol concentration would be similar, but the formation of side products, which are related to the flavor of the beverages, would be different among the strains.

Table 1. Fermentation results by using different yeast strains. Fermentation conditions: $\mathrm{T}=30^{\circ} \mathrm{C}$, $200 \mathrm{rpm}, \mathrm{pH}=5.5$, time $=24 \mathrm{~h}$.

\begin{tabular}{cccc}
\hline Fermentation Parameter & HG-1 & C-70 & M-1 \\
\hline $\begin{array}{c}\text { Yeast cell viability } \\
\text { (Log CFU/mL) }\end{array}$ & $1.89 \pm 0.05^{\mathrm{a}}$ & $1.69 \pm 0.12^{\mathrm{b}}$ & $2.03 \pm 0.01^{\mathrm{a}}$ \\
$\begin{array}{c}\text { Carbohydrate conversion } \\
\text { Ethanol concentration } \\
(\mathrm{g} / \mathrm{L})\end{array}$ & 95.6 & 95.6 & 95.5 \\
$\begin{array}{c}\text { Fusel alcohol concentration } \\
(\mathrm{g} / \mathrm{L})\end{array}$ & $80.06 \pm 3.88^{\mathrm{a}}$ & $74.87 \pm 9.70^{\mathrm{a}}$ & $77.85 \pm 4.91^{\mathrm{a}}$ \\
\hline
\end{tabular}

Different letters indicate significant differences among yeast strains $(p<0.05)$.

It is well known that 3-methyl-1-butanol is one of the main harsher impurities during SR $[3,10]$. Hence, HG-1 yeast strain was selected to investigate the effect of pretreatment and fermentation conditions on the amount of fusel alcohol since it allows a reduction in the amount of 3-methyl-1-butanol by at least 30\%. In the upcoming section, we explore the effect of pretreatment and the main fermentation variables, such as temperature, $\mathrm{pH}$, and stirring.

\subsection{Effect of Pretreatment and Fermentation Conditions}

The effect of hydrolysis conditions and fermentation parameters, such as $\mathrm{pH}$, temperature, and stirring, were carried out by using a Box-Behnken design model. Tables 2 and 3 show the results for yeast viability, carbohydrates conversion, ethanol, and fusel alcohol concentration for the hydrothermal and acid hydrolysis pretreatment conditions, respectively.

Table 4 shows the statistical results of the assessed factors, i.e., $\mathrm{pH}(\mathrm{A})$, stirring (B), temperature (C), and hydrolysis (D), over several response variables (i.e., yeast cell viability, carbohydrates conversion, ethanol, 1-propanol, 2-methyl-1-propanol, 3-methyl-1-butanol, and acetic acid). Mostly all response variables were adjusted to a quadratic model (Equation (1)) $(p<0.01$ and not significant lack of fits, $p>0.05)$. However, the conversion of both acetic acid and HMF showed a significant lack of fit $(p<0.05)$, indicating that those models have a poor prediction ability. Despite the significant lack of fit, the difference between the adjusted and predicted $\mathrm{R}^{2}$ was higher than 0.2 . Hence, those models represent the response surface well and could be used to give insights on the effect of the factors over both the acetic acid and HMF conversion during fermentation [36,37]. Furthermore, the $\mathrm{R}^{2}$ for all the response variables was higher than 0.80 . Therefore, the assessed factors (i.e., $\mathrm{pH}$, stirring, temperature, and hydrolysis) can explain $80 \%$ of the variability of the response variables. Table 5 shows the $\beta$ coefficients of the quadratic response shown in Equation (1). The sign of the $\beta$ coefficients indicates whether the effect of the factor is negative or positive [16]. 


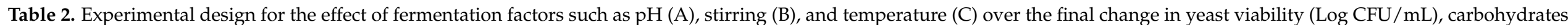

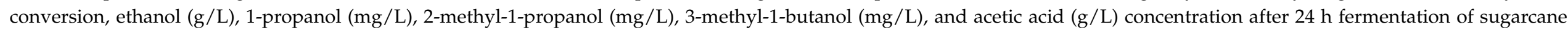
press-mud by using a S. cerevisiae strain (Ethanol Red, Fermentis ${ }^{\circledR}$ ). Hydrolysis conditions: no $\mathrm{HNO}_{3}$ addition; $130{ }^{\circ} \mathrm{C}, 1 \mathrm{~h}$.

\begin{tabular}{|c|c|c|c|c|c|c|c|c|c|c|c|c|c|c|c|c|c|}
\hline \multirow{3}{*}{ Run } & \multirow{3}{*}{$\begin{array}{l}\mathrm{A}: \\
\mathrm{pH}\end{array}$} & \multirow{3}{*}{$\begin{array}{c}\text { B: } \\
\text { Stirring } \\
\text { (rpm) }\end{array}$} & \multirow{3}{*}{$\begin{array}{c}\mathrm{C}: \\
\mathrm{T}\left({ }^{\circ} \mathrm{C}\right)\end{array}$} & \multirow{2}{*}{\multicolumn{2}{|c|}{$\begin{array}{c}\Delta \text { Yeast } \\
\text { Viability } \\
\text { (Log CFU } / \mathrm{mL})\end{array}$}} & \multirow{2}{*}{\multicolumn{2}{|c|}{$\begin{array}{l}\text { Carbohydrates } \\
\text { Conversion }\end{array}$}} & \multirow{2}{*}{\multicolumn{2}{|c|}{$\begin{array}{c}\text { Ethanol } \\
(\mathrm{g} / \mathrm{L})\end{array}$}} & \multicolumn{6}{|c|}{ Fusel Alcohol (mg/L) } & \multirow{2}{*}{\multicolumn{2}{|c|}{$\begin{array}{c}\text { Acetic Acid } \\
(\mathrm{g} / \mathrm{L})\end{array}$}} \\
\hline & & & & & & & & & & \multicolumn{2}{|c|}{ 1-Propanol } & \multicolumn{2}{|c|}{ 2M1P } & \multicolumn{2}{|c|}{ 3M1B } & & \\
\hline & & & & Exp. & Mod. & Exp. & Mod. & Exp. & Mod. & Exp. & Mod. & Exp. & Mod. & Exp. & Mod. & Exp. & Mod. \\
\hline 1 & 6.5 & 100 & 30 & 2.10 & 2.14 & 0.97 & 1.00 & 93.52 & 91.84 & 96.95 & 90.29 & 149.23 & 165.41 & 283.19 & 305.66 & 4.06 & 3.98 \\
\hline 2 & 6.5 & 200 & 25 & 2.37 & 2.40 & 0.98 & 0.99 & 94.22 & 97.80 & 89.42 & 90.41 & 214.82 & 194.86 & 369.13 & 342.93 & 3.88 & 4.00 \\
\hline 3 & 5.5 & 0 & 30 & 1.97 & 2.02 & 0.97 & 0.92 & 93.65 & 91.81 & 89.56 & 88.15 & 148.44 & 143.36 & 287.24 & 269.93 & 3.89 & 3.78 \\
\hline 7 & 5.5 & 200 & 20 & 2.09 & 2.10 & 0.51 & 0.52 & 66.13 & 61.61 & 49.15 & 47.21 & 89.19 & 100.12 & 148.78 & 161.37 & 5.56 & 5.33 \\
\hline 8 & 5.5 & 100 & 25 & 2.29 & 2.28 & 0.98 & 0.93 & 96.83 & 92.71 & 96.13 & 80.77 & 203.08 & 156.43 & 355.53 & 275.38 & 3.87 & 3.97 \\
\hline 14 & 4.5 & 0 & 25 & 1.97 & 2.06 & 0.95 & 0.94 & 91.92 & 90.36 & 78.01 & 73.51 & 129.68 & 135.43 & 230.68 & 237.09 & 3.87 & 3.96 \\
\hline 19 & 5.5 & 100 & 25 & 2.23 & 2.28 & 0.95 & 0.93 & 91.79 & 92.71 & 82.03 & 80.77 & 155.21 & 156.43 & 277.96 & 275.38 & 3.94 & 3.97 \\
\hline 24 & 5.5 & 100 & 25 & 2.35 & 2.28 & 0.95 & 0.93 & 92.45 & 92.71 & 72.37 & 80.77 & 168.90 & 156.43 & 286.27 & 275.38 & 3.91 & 3.97 \\
\hline 26 & 4.5 & 200 & 25 & 1.87 & 1.89 & 0.96 & 0.96 & 85.94 & 90.90 & 65.28 & 69.67 & 130.18 & 135.21 & 229.34 & 239.40 & 3.81 & 3.92 \\
\hline 28 & 6.5 & 0 & 25 & 2.47 & 2.40 & 0.94 & 0.94 & 89.02 & 89.66 & 77.33 & 84.07 & 157.28 & 161.69 & 257.25 & 268.50 & 3.99 & 3.96 \\
\hline 29 & 6.5 & 100 & 20 & 2.10 & 2.09 & 0.60 & 0.58 & 59.24 & 59.74 & 46.01 & 45.86 & 80.57 & 96.60 & 118.33 & 145.28 & 6.44 & 5.41 \\
\hline 30 & 5.5 & 0 & 20 & 2.34 & 2.33 & 0.36 & 0.48 & 44.70 & 47.60 & 31.67 & 36.14 & 65.32 & 75.47 & 91.28 & 111.38 & 6.45 & 5.40 \\
\hline 31 & 4.5 & 100 & 30 & 1.63 & 1.50 & 0.94 & 1.01 & 88.22 & 91.18 & 67.60 & 72.41 & 107.01 & 113.36 & 200.51 & 224.73 & 3.92 & 3.82 \\
\hline 32 & 5.5 & 200 & 30 & 2.06 & 2.08 & 0.93 & 0.95 & 90.38 & 86.49 & 77.72 & 79.59 & 132.43 & 151.66 & 272.66 & 296.67 & 3.88 & 3.85 \\
\hline
\end{tabular}

2M1P: 2-methyl-1-propanol; 3M1B: 3-methyl-1-butanol; Exp: experimental data; Mod: model-predicted data. 


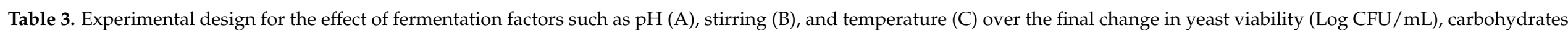

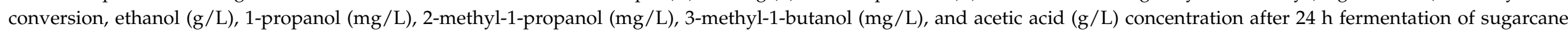
press-mud by using a S. cerevisiae strain (Ethanol Red, Fermentis ${ }^{\circledR}$ ). Hydrolysis conditions: $16 \mathrm{~g} \mathrm{HNO}_{3} / \mathrm{kg}$ dry solid; $130{ }^{\circ} \mathrm{C}, 1 \mathrm{~h}$.

\begin{tabular}{|c|c|c|c|c|c|c|c|c|c|c|c|c|c|c|c|c|c|}
\hline \multirow{3}{*}{ Run } & \multirow{3}{*}{$\begin{array}{l}\text { A: } \\
\text { pH }\end{array}$} & \multirow{3}{*}{$\begin{array}{c}\text { B: } \\
\text { Stirring } \\
(\mathrm{rpm})\end{array}$} & \multirow{3}{*}{$\begin{array}{c}\mathrm{C}: \\
\mathrm{T}\left({ }^{\circ} \mathrm{C}\right)\end{array}$} & \multirow{2}{*}{\multicolumn{2}{|c|}{$\begin{array}{c}\Delta \text { Yeast } \\
\text { Viability } \\
(\text { Log CFU/mL) }\end{array}$}} & \multirow{2}{*}{\multicolumn{2}{|c|}{$\begin{array}{c}\text { Carbohydrates } \\
\text { Conversion }\end{array}$}} & \multirow{2}{*}{\multicolumn{2}{|c|}{$\begin{array}{c}\text { Ethanol } \\
\text { (g/L) }\end{array}$}} & \multicolumn{6}{|c|}{ Fusel Alcohol (mg/L) } & \multirow{2}{*}{\multicolumn{2}{|c|}{$\begin{array}{l}\text { Acetic Acid } \\
\text { (g/L) }\end{array}$}} \\
\hline & & & & & & & & & & \multicolumn{2}{|c|}{ 1-Propanol } & \multicolumn{2}{|c|}{ 2M1P } & \multicolumn{2}{|c|}{ 3M1B } & & \\
\hline & & & & Exp. & Mod. & Exp. & Mod. & Exp. & Mod. & Exp. & Mod. & Exp. & Mod. & Exp. & Mod. & Exp. & Mod. \\
\hline 4 & 6.5 & 100 & 30 & 2.09 & 2.06 & 0.91 & 0.91 & 90.06 & 83.79 & 68.47 & 68.72 & 93.92 & 107.06 & 176.40 & 191.84 & 4.48 & 3.58 \\
\hline 5 & 6.5 & 200 & 25 & 1.99 & 1.97 & 0.93 & 0.89 & 95.90 & 93.01 & 91.60 & 89.70 & 147.41 & 136.70 & 271.38 & 251.23 & 3.98 & 2.86 \\
\hline 6 & 5.5 & 0 & 30 & 1.86 & 1.85 & 1.01 & 0.91 & 94.83 & 87.64 & 95.62 & 80.84 & 152.55 & 128.11 & 256.69 & 218.79 & 3.91 & 3.17 \\
\hline 9 & 5.5 & 200 & 20 & 2.05 & 1.95 & 0.94 & 0.89 & 94.36 & 95.02 & 87.07 & 82.30 & 131.86 & 107.13 & 228.36 & 199.84 & 4.03 & 3.16 \\
\hline 10 & 5.5 & 100 & 25 & 1.77 & 1.79 & 0.46 & 0.37 & 51.92 & 42.41 & 31.64 & 21.37 & 64.96 & 38.03 & 101.28 & 50.36 & 5.66 & 4.19 \\
\hline 12 & 5.5 & 100 & 25 & 2.27 & 2.14 & 0.81 & 0.83 & 91.10 & 86.92 & 79.47 & 74.39 & 116.24 & 122.47 & 205.53 & 213.50 & 4.34 & 3.13 \\
\hline 13 & 4.5 & 0 & 25 & 1.92 & 1.94 & 0.91 & 0.89 & 95.21 & 95.83 & 80.40 & 82.85 & 129.38 & 123.59 & 223.42 & 218.95 & 4.38 & 2.82 \\
\hline 15 & 5.5 & 100 & 25 & 2.33 & 2.14 & 0.88 & 0.83 & 89.99 & 86.92 & 78.31 & 74.39 & 134.53 & 122.47 & 215.61 & 213.50 & 4.20 & 3.13 \\
\hline 16 & 5.5 & 100 & 25 & 2.09 & 2.14 & 0.83 & 0.83 & 86.44 & 86.92 & 78.46 & 74.39 & 124.64 & 122.47 & 201.50 & 213.50 & 4.22 & 3.13 \\
\hline 17 & 4.5 & 200 & 25 & 1.41 & 1.53 & 0.98 & 1.03 & 90.89 & 96.93 & 78.50 & 84.12 & 99.44 & 104.00 & 196.15 & 195.91 & 4.11 & 3.28 \\
\hline 18 & 6.5 & 0 & 25 & 1.86 & 1.90 & 0.26 & 0.35 & 41.12 & 46.81 & 32.16 & 40.28 & 49.55 & 68.42 & 74.47 & 107.71 & 5.39 & 4.28 \\
\hline 20 & 6.5 & 100 & 20 & 1.95 & 2.14 & 0.69 & 0.83 & 81.96 & 86.92 & 59.89 & 74.39 & 79.77 & 122.47 & 160.73 & 213.50 & 4.61 & 3.13 \\
\hline 21 & 5.5 & 0 & 20 & 1.64 & 2.17 & 0.43 & 0.33 & 25.64 & 29.50 & 17.67 & 13.25 & 41.15 & 22.49 & 57.03 & 33.07 & 5.87 & 4.72 \\
\hline 22 & 4.5 & 100 & 30 & 2.05 & 2.14 & 0.82 & 0.83 & 86.35 & 86.92 & 70.97 & 74.39 & 88.58 & 122.47 & 175.84 & 213.50 & 4.42 & 3.13 \\
\hline 23 & 5.5 & 200 & 30 & 2.20 & 2.13 & 0.81 & 0.81 & 88.55 & 92.79 & 79.16 & 82.42 & 154.24 & 155.30 & 260.09 & 264.44 & 4.33 & 2.63 \\
\hline
\end{tabular}

2M1P: 2-methyl-1-propanol; 3M1B: 3-methyl-1-butanol; Exp: experimental data; Mod: model-predicted data. 
Table 4. ANOVA results for the adjusted variables to response optimization in response to $\mathrm{pH}(\mathrm{A})$, stirring (B), temperature (C), and hydrolysis (D).

\begin{tabular}{|c|c|c|c|c|c|c|c|c|c|c|}
\hline & $\begin{array}{l}\text { Yeast Cell Viability } \\
\text { (Log CFU/mL) }\end{array}$ & $\begin{array}{c}\text { Carbohydrates } \\
\text { Conversion }\end{array}$ & $\begin{array}{c}\text { Ethanol } \\
(\mathrm{g} / \mathrm{L})\end{array}$ & $\begin{array}{c}1 \mathrm{P} \\
(\mathrm{mg} / \mathrm{L})\end{array}$ & $\begin{array}{l}2 \mathrm{M} 1 \mathrm{P} \\
(\mathrm{mg} / \mathrm{L})\end{array}$ & $\begin{array}{l}\text { 3M1B } \\
\text { (mg/L) }\end{array}$ & $\begin{array}{c}\text { Acetic Acid } \\
(\mathrm{g} / \mathrm{L})\end{array}$ & $\begin{array}{c}\text { Fusel } \\
\text { Alcohol (mg/L) }\end{array}$ & $\begin{array}{l}\text { Acetic Acid } \\
\text { Conversion }\end{array}$ & $\begin{array}{c}\text { HMF } \\
\text { Conversion }\end{array}$ \\
\hline Model & $<0.0001$ & $<0.0001$ & $<0.0001$ & $<0.0001$ & $<0.0001$ & $<0.0001$ & $<0.0001$ & $<0.0001$ & $<0.0001$ & $<0.0001$ \\
\hline A & $<0.0001$ & 0.1845 & 0.4015 & 0.1818 & 0.0391 & 0.0546 & 0.1005 & 0.0482 & $<0.0001$ & 0.0845 \\
\hline B & 0.0618 & 0.5273 & 0.0315 & 0.0481 & 0.0366 & 0.0142 & 0.0433 & 0.0184 & 0.0298 & 0.1461 \\
\hline $\mathrm{C}$ & 0.0346 & $<0.0001$ & $<0.0001$ & $<0.0001$ & $<0.0001$ & $<0.0001$ & $<0.0001$ & $<0.0001$ & $<0.0001$ & $<0.0001$ \\
\hline $\mathrm{D}$ & 0.0009 & 0.0006 & 0.0040 & 0.0464 & 0.0006 & 0.0001 & $<0.0001$ & 0.0003 & 0.0086 & 0.0326 \\
\hline $\mathrm{AB}$ & 0.2459 & 0.7727 & 0.3120 & 0.4213 & 0.3409 & 0.1917 & 0.7394 & 0.2416 & 0.9835 & 0.8812 \\
\hline $\mathrm{AC}$ & 0.0066 & 0.6597 & 0.5124 & 0.7221 & 0.6009 & 0.6197 & 0.3434 & 0.6108 & 0.2882 & 0.0154 \\
\hline $\mathrm{AD}$ & 0.0356 & 0.1175 & 0.7374 & 0.0410 & 0.1950 & 0.1408 & 0.2147 & 0.1219 & 0.6181 & 0.8467 \\
\hline $\mathrm{BD}$ & 0.7478 & 0.6808 & 0.5310 & 0.0838 & 0.3901 & 0.5211 & 0.0459 & 0.3723 & 0.6477 & 0.8472 \\
\hline $\mathrm{CD}$ & 0.3643 & 0.1293 & 0.0005 & 0.0663 & 0.4970 & 0.8300 & 0.7530 & 0.5425 & 0.0220 & 0.0560 \\
\hline $\mathrm{A}^{2}$ & 0.0002 & 0.0725 & 0.6141 & 0.5286 & 0.6470 & 0.4182 & 0.5146 & 0.3863 & 0.9374 & 0.6569 \\
\hline $\mathrm{B}^{2}$ & 0.0678 & 0.4034 & 0.4283 & 0.8497 & 0.6165 & 0.5780 & 0.4176 & 0.6079 & 0.6923 & 0.7607 \\
\hline$C^{2}$ & $<0.0001$ & $<0.0001$ & $<0.0001$ & $<0.0001$ & $<0.0001$ & $<0.0001$ & $<0.0001$ & $<0.0001$ & $<0.0001$ & $<0.0001$ \\
\hline Lack of fit & 0.8412 & 0.0877 & 0.0996 & 0.7494 & 0.5588 & 0.5636 & 0.1143 & 0.6233 & 0.0007 & 0.0002 \\
\hline$R^{2}$ & 0.8877 & 0.9321 & 0.9588 & 0.9084 & 0.8181 & 0.8719 & 0.9646 & 0.8700 & 0.9271 & 0.9641 \\
\hline $\mathrm{R}^{2}$ adjusted & 0.8108 & 0.8880 & 0.9321 & 0.8489 & 0.6998 & 0.7886 & 0.9391 & 0.7855 & 0.8745 & 0.9366 \\
\hline
\end{tabular}

Green filled $(p<0.0001)$, blue filled $(0.0001<p<0.05)$, orange filled $(0.05<p<0.10)$, dark gray $(p>0.10)$

Table 5. Equation terms of coded factors. $\mathrm{pH}(\mathrm{A})$, stirring (B), temperature (C), hydrolysis (D).

\begin{tabular}{|c|c|c|c|c|c|c|c|c|c|c|c|c|c|c|}
\hline & Int. & A & B & $\mathrm{C}$ & D & $\mathrm{AB}$ & AC & AD & BC & BD & $\mathrm{CD}$ & $A^{2}$ & $\mathbf{B}^{2}$ & $\mathrm{C}^{2}$ \\
\hline YCV (CFU/mL) & 2.21 & 0.15 & -0.05 & -0.06 & 0.07 & 0.04 & 0.11 & 0.06 & 0.07 & 0.01 & -0.03 & -0.16 & 0.07 & -0.21 \\
\hline XG $(\%)$ & 0.88 & -0.02 & 0.01 & 0.25 & 0.05 & 0.01 & -0.01 & 0.03 & -0.00 & 0.01 & -0.03 & 0.05 & -0.02 & -0.19 \\
\hline$E(g / L)$ & 89.81 & 1.11 & 3.00 & 22.6 & 2.89 & 1.90 & -1.22 & 0.44 & -4.83 & -0.82 & -5.33 & 0.92 & -1.45 & -19.4 \\
\hline 2M1P (mg/L) & 139.4 & 13.3 & 13.5 & 34.0 & 17.0 & 8.35 & 4.55 & 8.12 & -4.09 & -5.32 & -4.19 & -3.88 & 4.24 & -43.0 \\
\hline $3 \mathrm{M} 1 \mathrm{~B}(\mathrm{mg} / \mathrm{L})$ & 244.4 & 19.2 & 25.3 & 75.5 & 30.9 & 18.03 & 6.73 & 14.47 & -5.81 & -6.16 & -2.03 & -10.8 & 7.36 & -72.9 \\
\hline $\mathrm{AA}(\mathrm{g} / \mathrm{L})$ & 4.129 & 0.07 & -0.09 & -0.76 & -0.16 & 0.019 & 0.06 & -0.05 & 0.03 & 0.09 & -0.02 & 0.04 & -0.06 & 0.67 \\
\hline FA (mg/L) & 461.4 & 35.6 & 43.5 & 135 & 51.1 & 28.93 & 12.4 & 27.38 & -14.8 & -15.4 & -10.5 & -16.6 & 12.2 & -135 \\
\hline XHMF & 0.990 & 0.016 & 0.01 & 0.14 & 0.01 & 0.002 & -0.04 & 0.002 & -0.02 & 0.00 & -0.02 & 0.01 & 0.00 & -0.14 \\
\hline
\end{tabular}

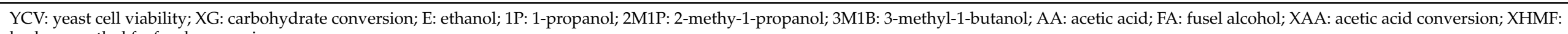
hydroxymethyl furfural conversion. 


\subsubsection{Inhibitory Compounds}

Previous studies showed that two inhibitory compounds (i.e., acetic acid and HMF), were mainly detected during pretreatment of sugarcane press-mud [7]. Table 6 shows that the initial concentration of acetic acid was $5.64 \pm 1.15 \mathrm{~g} / \mathrm{L}$ and $6.51 \pm 0.74 \mathrm{~g} / \mathrm{L}$ under hydrothermal and acid hydrolysis, respectively. Besides, the initial concentration of HMF was $3.40 \pm 1.16 \mathrm{~g} / \mathrm{L}$ under hydrothermal conditions. Whilst under acidic conditions, the HMF concentration was $6.64 \pm 1.16 \mathrm{~g} \cdot \mathrm{L}^{-1}$, and ANOVA showed that there was not a significant difference between pretreatment conditions on the production of fermentation inhibitors $(p<0.05)$. However, fermentation conditions affected the uptake of acetic acid and HMF, as shown in Table 4.

Table 6. Initial composition of the hydrolysates before fermentation. Pretreatment conditions: $\mathrm{T}=130{ }^{\circ} \mathrm{C}, 1 \mathrm{~h}$.

\begin{tabular}{ccc}
\hline Component & Hydrothermal & Acid Hydrolysis \\
\hline Carbohydrates (GE/L) & $146.10 \pm 17.47^{\mathrm{a}}$ & $134.45 \pm 38.60^{\mathrm{a}}$ \\
Acetic acid (g/L) & $5.64 \pm 1.15^{\mathrm{a}}$ & $6.51 \pm 0.74^{\mathrm{a}}$ \\
HMF (g/L) & $3.40 \pm 1.16^{\mathrm{a}}$ & $6.64 \pm 2.24^{\mathrm{a}}$ \\
\hline Different letters indicate significant differences among yeast strains $(p<0.05)$. &
\end{tabular}

Table 4 shows that acetic acid conversion was influenced by all the main effects (i.e., $\mathrm{pH}(\mathrm{A})$, stirring (B), temperature (C), and pretreatment (D)). Besides, the interaction between fermentation temperature and pretreatment condition $(C D)$ and the square of the temperature $\left(C^{2}\right)$ had a significant effect on the acetic acid conversion. Table 5 portrays that among the significant variables, $C^{2}$ had the highest effect $(\beta=-0.14)$, followed by temperature $(\mathrm{C})(\beta=0.15)$ and $\mathrm{pH}(\mathrm{A})(\beta=-0.08)$. The negative effect of the square temperature indicates that acetic acid conversion reached a maximum [38]. Figure 1a shows that the maximum acetic acid conversion was $33 \%$ at $26^{\circ} \mathrm{C}$, regardless of the pretreatment conditions when sugarcane press-mud was fermented at a $\mathrm{pH}$ of 5.5 and $100 \mathrm{rpm}$. Besides, the effect of pretreatment on acetic acid conversion was clear when fermentation was carried out at $20^{\circ} \mathrm{C}$. Rising fermentation temperature hindered the effect of pretreatment conditions. An opposite behavior was observed by Shang et al. [39] who reported that the higher the temperature was, the higher the accumulation of acetic acid and the lower the conversion during fermentation of lychee juice and using S. cerevisiae 2137 strain would be. Differences were associated with the yeast strain and medium nutrients that could affect the acetic acid uptake [40]. Herein, the initial concentration of carbohydrates was $146.10 \pm 17.47 \mathrm{GE} / \mathrm{L}$ and $134.45 \pm 38.60 \mathrm{GE} / \mathrm{L}$ after the pretreatment of sugarcane pressmud under hydrothermal and acid hydrolysis conditions, respectively, as shown in Table 6. In addition, the initial concentration of acetic acid was above $5.0 \mathrm{~g} / \mathrm{L}$, regardless of the pretreatment conditions. Furthermore, the culture was supplemented with yeast extract, peptone, $\mathrm{MgSO}_{4} \cdot 7 \mathrm{H}_{2} \mathrm{O}, \mathrm{Ca}_{3}\left(\mathrm{PO}_{4}\right)_{2}$, and $\left(\mathrm{NH}_{4}\right)_{2} \mathrm{SO}_{4}$. Herein, two carbon sources were identified: sugar and acetic acid. Different enzymes such as PDC (pyruvate decarboxylase), ADH (alcohol dehydrogenase), ALDH (acetaldehyde dehydrogenase), ACS (acetyl-CoA synthase), and ILC (isocitrate lyase) act during the metabolism of acetic acid [39]. In the presence of acetic acid, ALDH, which catalyzes the oxidation of acetaldehyde into acetic acid, is suppressed, while the activity of PDC and ADH increases. PDC catalyzes the decarboxylation of pyruvate into acetaldehyde and $\mathrm{ADH}$ catalyzes the reduction of acetaldehyde into ethanol [39]. In addition, acetic acid is also converted into acetyl CoA by ACS. Acetyl CoA could be converted into acetaldehyde via EutE using cytosolic NADH [41]. The high availability of acetaldehyde might result in a larger concentration of ethanol. 

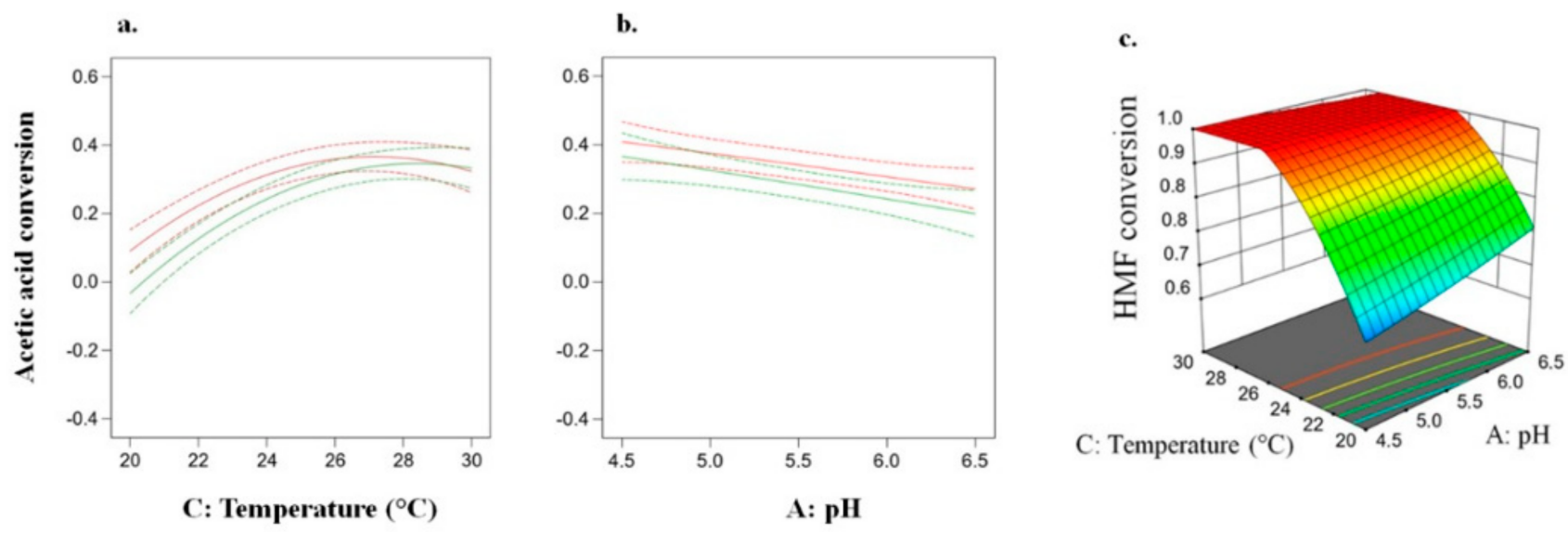

Figure 1. (a) Effect of the fermentation temperature and pretreatment condition on the acetic acid conversion (green $=$ acid hydrolysis, red = hydrothermal), fermentation conditions: $\mathrm{pH}=5.5,100 \mathrm{rpm}, \mathrm{t}=24 \mathrm{~h}$; (b) Effect of the fermentation $\mathrm{pH}$ and pretreatment condition on the acetic acid conversion (green = acid hydrolysis, red = hydrothermal), fermentation conditions: $\mathrm{pH}=5.5,100 \mathrm{rpm}, \mathrm{t}=24 \mathrm{~h}$; (c) Effect of the fermentation $\mathrm{pH}(\mathrm{A})$ and temperature (C) on the HMF conversion.

Figure 2 shows the Spearman correlation coefficient (RHO) among response variables. RHO is employed when there is a mixture that is normally and non-normally distributed [10,42]. According to the Shapiro-Wilk test, shown in Figure 2, the following variables were not normally distributed: carbohydrate conversion (CC), ethanol concentration (ET), 1-propanol concentration (1P), acetic acid concentration (AA), acetic acid conversion, and HMF conversion $(p<0.05)$. On the other hand, yeast, 2-methyl-1-propanol concentration (2M1P), 3-methyl-1-butanol concentration, and total fusel alcohol concentration were normally distributed. Here, a positive correlation between ethanol and acetic acid conversion was observed $(\mathrm{RHO}=0.674)$. The positive correlation showed that acetic acid could act as substrate to produce ethanol [42], as explained above. The consumption of acetic acid along with an increment on the ethanol production was also observed by Wei et al. [43] during the co-fermentation of synthetic culture based on glucose and using S. cerevisiae strain at $30^{\circ} \mathrm{C}$.

\begin{tabular}{|c|c|c|c|c|c|c|c|c|c|c|}
\hline Response variable & Yeast & $\mathrm{CC}$ & ET & $1 P$ & 2MlP & 3M1B & & AA & AAC & HMFC \\
\hline Yeast & .000 & 0.165 & 0.282 & 0.314 & 0.583 & 0.489 & 0.489 & -0.184 & -0.023 & 0.308 \\
\hline Carbohydrate conversion (CC) & & 1.000 & 0.813 & 0.795 & 0.807 & 0.836 & 0.842 & -0.865 & 0.725 & 0.844 \\
\hline Ethanol (ET) & & & 1.000 & 0.935 & 0.809 & 0.846 & 0.877 & -0.707 & 0.674 & 0.805 \\
\hline 1-Propanol (1P) & & & & 1.000 & 0.823 & 0.849 & 0.886 & -0.670 & 0.584 & 0.740 \\
\hline 2-methyl-1-propanol (2M1P) & & & & & 1.000 & 0.966 & 0.973 & -0.803 & 0.526 & 0.829 \\
\hline 3-methyl-1-butanol (3M1B) & & & & & & 1.000 & 0.991 & -0.857 & 0.557 & 0.831 \\
\hline Fusel alcohol (FA) & & & & & & & 1.000 & -0.823 & 0.556 & 0.832 \\
\hline Acetic acid (AA) & & & & & & & & 1.000 & 0.740 & -0.808 \\
\hline Acetic acid conversion (AAC) & & & & & & & & & 1.000 & 0.752 \\
\hline HMF conversion (HMFC) & & & & & & & & & & 1.000 \\
\hline Normality test (Shapiro Wil & 0.392 & 0.000 & 0.000 & 0.003 & 0.535 & 0.475 & 0.347 & 0.000 & 0.000 & 0.000 \\
\hline Homogeneity test (Levene's test) & 0.877 & 0.512 & 0.139 & 0.305 & 0.751 & 0.408 & 0.691 & 0.103 & 0.503 & 0.171 \\
\hline
\end{tabular}

\begin{tabular}{|ccccccccccccccccccccc|}
\hline-1.0 & -0.9 & -0.8 & -0.7 & -0.6 & -0.5 & -0.4 & -0.3 & -0.2 & -0.1 & 0.0 & 0.1 & 0.2 & 0.3 & 0.4 & 0.5 & 0.6 & 0.7 & 0.8 & 0.9 & 1.0 \\
\hline I \\
No correlation
\end{tabular}
$\begin{aligned} & \text { Negative very strong } \\
& \text { correlation }\end{aligned}$

Figure 2. Spearman correlation coefficient matrix $(p<0.05)$ among the response variables tested during the fermentation of sugarcane press-mud, as well as $p$ values for the normality test and homogeneity test. 
Concerning the effect of $\mathrm{pH}$, Figure $1 \mathrm{~b}$ shows that at $\mathrm{pH}=4.0$, acetic acid conversion was about $40 \%$. However, an increment in the $\mathrm{pH}$ resulted in a drop off of acetic acid conversion. The higher acetic acid conversion at low $\mathrm{pH}$ was associated with the amount of undissociated forms of acetic acid that permeates the yeast membrane. The fraction of undissociated forms is determined by the $\mathrm{pKa}$, whose value is 4.6. This means that acetic acid dissociates when $\mathrm{pH}>\mathrm{pKa}$ and hence, higher undissociated forms of acetic acid are found at low $\mathrm{pH}$ values. For instance, at $\mathrm{pH}=4.0$, the fraction of undissociated acid is $64 \%$ [44]. The permeation of undissociated forms of acetic acid acidifies the cytosol, reduces the metabolic activity during fermentation, and under extreme conditions, inhibits the yeast activity [41]. Yang et al. [44] showed when acetic acid content was higher than $7.5 \mathrm{~g} / \mathrm{L}$ at a $\mathrm{pH}$ of 4.5, a considerable drop off was observed for the cell number during fermentation of glucose synthetic culture by using a commercial strain TG1348 of S. cerevisiae at $30^{\circ} \mathrm{C}$ without shaking. Herein, the maximum initial content of acetic acid was $6.51 \pm 0.74 \mathrm{~g} / \mathrm{L}$, as shown in Table 6, concluding that the amount of acetic acid present in the sugarcane press-mud hydrolysates did not have an inhibitory effect, since the RHO value between yeast cell viability and acetic acid conversion was -0.023 according to Figure 2.

Moreover, as shown in Table 4, HMF conversion was affected by the temperature (C) and the pretreatment (D). Besides, the interaction between the fermentation $\mathrm{pH}$ and temperature $(\mathrm{AC})$ and the square of temperature $\left(\mathrm{C}^{2}\right)$ had a significant effect on HMF conversion. According to Table 5, the highest effect was observed for the temperature, followed by $\mathrm{AC}$ and the pretreatment conditions, since the $\beta$ values were $-0.14,-0.04$, and 0.01 , respectively. The negative value of the $\beta$ coefficient for the squared term of temperature $\left(C^{2}\right)$, i.e., -0.14 , represents a maximum conversion of HMF. This result can be clearly observed in Figure $1 \mathrm{c}$, where at $27^{\circ} \mathrm{C}$, a maximum conversion of HMF was observed when sugarcane press-mud was fermented at a pH of 6.5. Akillioglu et al. [45] reported complete conversion of HMF by using ground malt, as raw material, and S. cerevisiae at $30^{\circ} \mathrm{C}$ for $24 \mathrm{~h}$. HMF is reduced by the enzymatic activity of alcohol dehydrogenase that is affected by fermentation parameters such as $\mathrm{pH}$ and temperature $[39,46,47]$. Besides, according to Figure 2, HMF conversion was better correlated with yeast cell viability than acetic acid conversion whose RHO were 0.308 and -0.023 , respectively. Hence, HMF had a stronger inhibition effect than acetic acid. The positive value of RHO indicated that the lower the HMF conversion was, the lower the yeast cell viability would be. Lee et al. [48] reported a similar result during the fermentation of lignocellulosic hydrolysates by using $S$. cerevisiae $\mathrm{K} 35$ strains at $30{ }^{\circ} \mathrm{C}$.

\subsubsection{Yeast Cell Viability}

The initial yeast cell viability for all the experiments was $6.56 \pm 0.13 \mathrm{Log} C F U / \mathrm{mL}$. According to statistical results described in Tables 4 and 5 , yeast cell viability was mainly affected $(p<0.05)$ by the interaction between $\mathrm{pH}$ and temperature (AC, in Table 4$)$, the interaction between $\mathrm{pH}$ and hydrolysis $(\mathrm{AD}$, in Table 4$)$ and the quadratic terms of both $\mathrm{pH}$ $\left(\mathrm{A}^{2}\right.$, in Table 4$)$ and temperature $\left(\mathrm{C}^{2}\right.$, in Table 4$)$. The $\beta$ coefficients depicted in Table 5 show that the square term of temperature $\left(C^{2}, \beta=-0.21\right)$ had the strongest influence, followed by the square term of $\mathrm{pH}\left(\mathrm{A}^{2}, \beta=-0.16\right)$, the interaction between $\mathrm{pH}$ and temperature ( $\mathrm{AC}, \beta=0.11$ ), and the interaction between $\mathrm{pH}$ and hydrolysis (AD, $\beta=0.06$ ). Figure $3 \mathrm{a}$ shows that there is a maximum yeast cell viability at $25^{\circ} \mathrm{C}$ and at a $\mathrm{pH}$ of 5.5 when sugarcane press-mud was pretreated under hydrothermal conditions. Thus, eliciting that significant effect of the square term of temperature reflects a maximum yeast cell viability at a specific temperature and $\mathrm{pH}$ value. A similar result was observed by Heard and Fleet [49] and Lu et al. [50]. Figure 3 shows an average increment of $2.14 \pm 0.22 \mathrm{Log} C F U / \mathrm{mL}$ and $1.97 \pm 0.23 \mathrm{Log} \mathrm{CFU} / \mathrm{mL}$ when sugarcane press-mud was pretreated under hydrothermal and acid hydrolysis conditions, respectively. A decrease in the yeast cell viability was observed at higher temperatures, possibly associated with the accumulation of ethanol that has a toxic effect on the yeast cell [50]. However, a successful adaptation of the microorganism is represented by an increment in almost 2 Log units [51]. Therefore, $S$. 
cerevisiae was fruitfully adapted, regardless of the pretreatment conditions, to the classical yeast medium formed by peptone, yeast extract, $\mathrm{MgSO}_{4} \cdot 7 \mathrm{H}_{2} \mathrm{O}, \mathrm{Ca}_{3}(\mathrm{PO} 4)_{2}$, and $\left(\mathrm{NH}_{4}\right)_{2} \mathrm{SO}_{4}$, where the carbon source was sugarcane press-mud, an agro-industrial residue with high content of fermentable sugars such as sucrose and glucose.

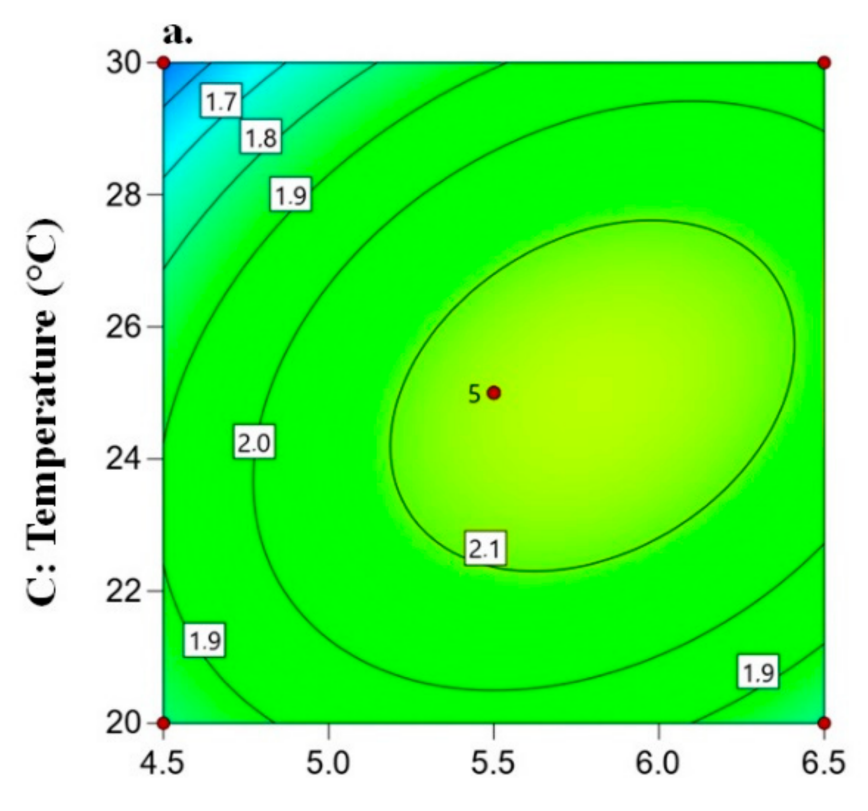

A: pH

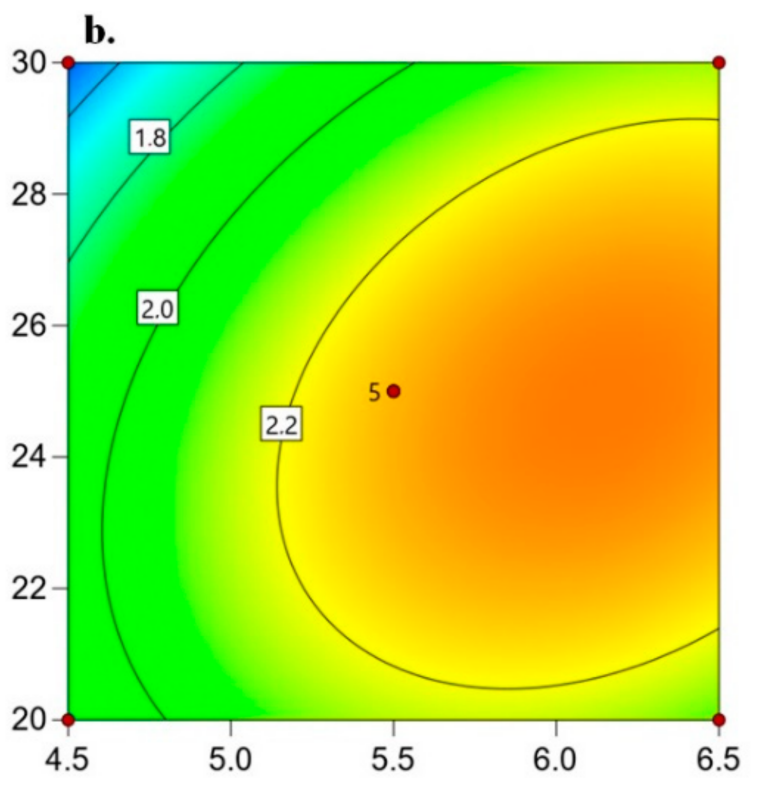

A: pH

Figure 3. Effect of temperature and $\mathrm{pH}$ on the yeast cell viability during fermentation after the pretreatment of sugarcane press-mud under (a) acid hydrolysis and (b) hydrothermal conditions. Fermentation conditions: stirring, 100 rpm; time, $24 \mathrm{~h}$.

\subsubsection{Carbohydrate Conversion}

Table 6 shows that the initial carbohydrate content was $146.10 \pm 17.47 \mathrm{GE} / \mathrm{L}$ and $134.45 \pm 38.60 \mathrm{GE} / \mathrm{L}$ after the pretreatment under hydrothermal and acid hydrolysis conditions, respectively. ANOVA depicted that there was not a significant difference between the carbohydrate content for both pretreatment conditions. This result agrees with previous results obtained during fermentation of sugarcane press-mud under different pretreatment conditions [7]. Sugarcane press-mud is a feedstock rich in carbohydrates (i.e., 22.95 wt.\% carbohydrates), as shown in Section 2.1. Sucrose is the main sugar present in sugarcane press-mud, as has been previously reported [7,52]. The content of fiber is low in comparison with the carbohydrate content. Indeed, fiber concentration in sugarcane press-mud is about $2.0 \mathrm{wt}$ \%, as shown in Section 2.1. Fiber refers to cellulose, hemicellulose, and lignin [53]. Under acid hydrolysis conditions, fiber fractions could decompose, thus producing hexoses such as glucose and galactose and pentoses such as xylose and arabinose [54]. However, due to the low content of fiber in comparison with other carbohydrates, the contribution of fermentable sugars from fiber decomposition will be negligible. The pretreatment of sugarcane press-mud increases the content of reducing sugar, such as glucose and fructose, to ease the fermentation process [7]. Under acid hydrolysis conditions, the amount of reducing sugar will be higher than under hydrothermal conditions due to the activity of $\mathrm{H}^{+}$. Figure 4 shows that carbohydrate conversion during fermentation at $20{ }^{\circ} \mathrm{C}$ of hydrolysates obtained through acid hydrolysis of sugarcane press-mud was higher than that observed after the fermentation of hydrolysates obtained through hydrothermal conditions. However, at temperatures above $25^{\circ} \mathrm{C}$, carbohydrate conversion was similar, regardless of the pretreatment condition. As described above, sucrose and glucose are the main sugars present in the hydrolysates from sugarcane press-mud, while sucrose must be converted into glucose and fructose by an invertase enzyme [55]. Glucose is directly metabolized by 
yeast through the glycolytic pathway [56]. Glycolytic enzymes such as phosphoglucose isomerase, phosphofructokinase, and pyruvate kinase are not strongly affected by temperature [57], while invertase activity is highly affected by temperature [58]. Therefore, at a higher glucose concentration and lower temperature, yeast will uptake carbohydrates faster than when there are at low glucose levels and high sucrose content. However, an increment on the fermentation temperature will rise the invertase activity, thus producing glucose and keeping similar carbohydrate conversion.

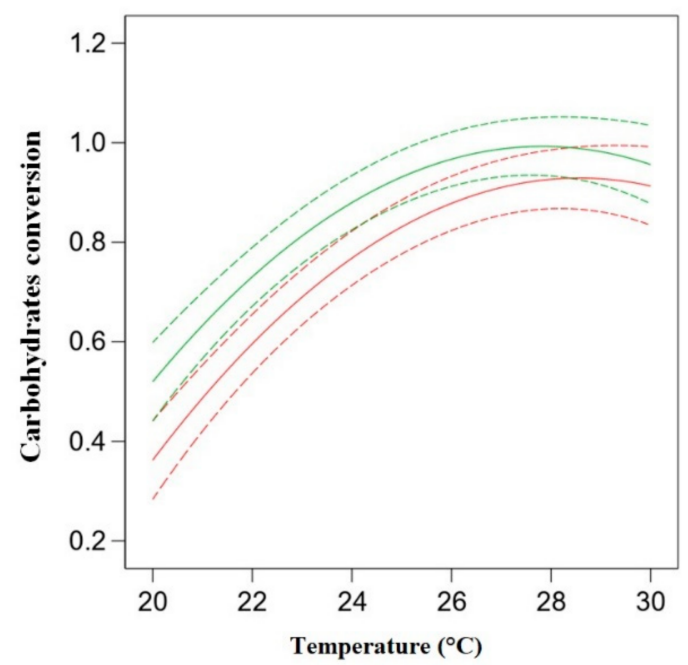

Figure 4. Effect of the fermentation temperature and pretreatment condition on the acetic acid conversion (green = acid hydrolysis, red = hydrothermal), fermentation conditions: $\mathrm{pH}=5.5,100 \mathrm{rpm}$, $\mathrm{t}=24 \mathrm{~h}$. Straight lines represent average value. Dashed lines represent lower and upper level with 95\% confidence level.

On the other hand, during fermentation of sugarcane press-mud, acetic acid and carbohydrates served as carbon source to yield ethanol. However, carbohydrates conversion was always higher than acetic acid. Those sugars are firstly uptaken by yeast, and after glucose exhaustion, acetic acid serves as a carbon source [59]. Figure 2 shows that carbohydrate conversion was strongly correlated with ethanol production since RHO was higher than 0.9. Carbohydrates are the main substrate employed by yeast to produce ethanol.

\subsubsection{Ethanol Concentration}

Ethanol is the main metabolite produced during fermentation of feedstocks with high content of hexoses. According to Table 4, the following main effects, interaction effects, and quadratic effects had a significant influenced on the ethanol concentration $(p<0.05)$ : stirring $(B)$, temperature $(C)$, pretreatment $(D)$, the interaction between stirring and fermentation temperature $(\mathrm{BC})$, the interaction between fermentation temperature and pretreatment conditions $(\mathrm{CD})$, and the square of temperature $\left(\mathrm{C}^{2}\right)$. Among them, $\mathrm{C}^{2}$ had the greatest influence, which is possibly ascribed to the enzyme activities that are sensitive to temperature [60]. Figure 5 shows that a maximum ethanol concentration was observed at $28^{\circ} \mathrm{C}$, regardless of the pretreatment conditions, which is within the optimum range temperature to carry out fermentation for free S. cerevisiae cells [61,62]. Moreover, at $20^{\circ} \mathrm{C}$, a clear effect of the pretreatment was observed. For instance, at said temperature, the ethanol concentration was 50 and $30 \mathrm{~g} / \mathrm{L}$ for the hydrolysates obtained under hydrothermal and acid hydrolysis conditions, respectively. However, an increment of the fermentation temperature hindered the effect of the pretreatment conditions. The outstanding effect of temperature on the ethanol concentration was associated with the activity of key enzymes that are involved in the central metabolism of $S$. cerevisiae $[50,63]$. 

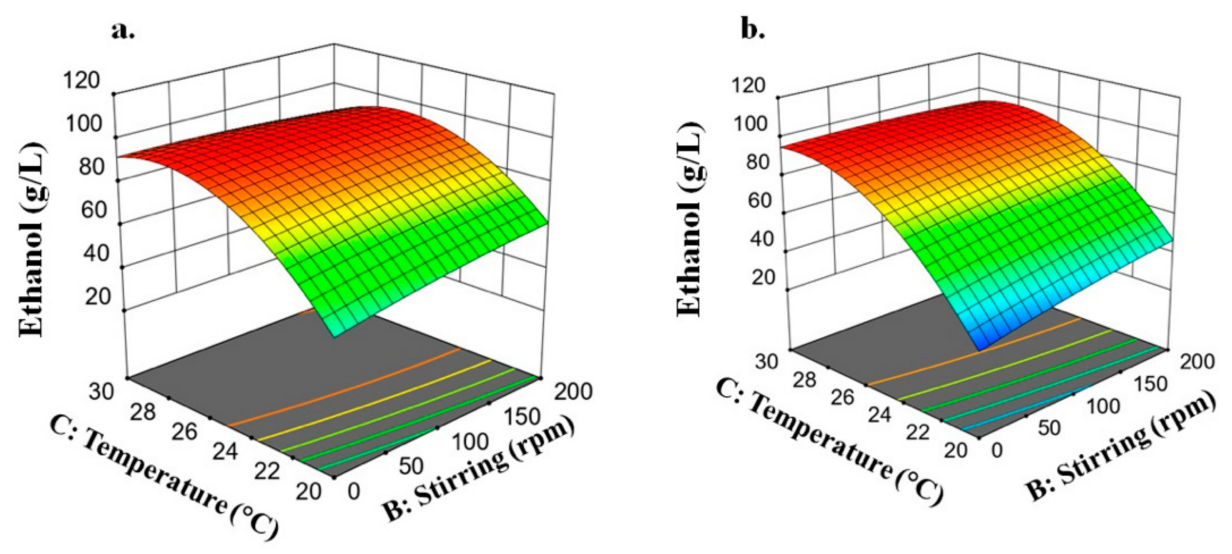

Figure 5. Effect of the temperature and stirring on the production of ethanol concentration during fermentation of hydrolysates obtained after pretreatment under (a) hydrothermal and (b) acid hydrolysis conditions. Fermentation $\mathrm{pH}=5.50$, fermentation time $=24 \mathrm{~h}$.

Concerning the effect of stirring, Figure $5 \mathrm{a}, \mathrm{b}$ shows that stirring had a more notable effect at $20^{\circ} \mathrm{C}$, since an increment of the stirring speed caused a rise in the ethanol concentration. Stirring is an important fermentation variable because it promotes homogeneity and allows contact between the free yeast cells, suspended in the fermentation culture, and the substrate [62]. Poor stirring leads to a low mass transfer coefficient and consequently affects fermentation performance [64]. At higher fermentation temperatures $\left(>26{ }^{\circ} \mathrm{C}\right)$, the effect of mechanical agitation is hampered, possibly ascribed to the formation of $\mathrm{CO}_{2}$. Under aerobic conditions and high glucose concentration, S. cerevisiae displays the Crabtree effect. This effect is associated with the use of both respiration and fermentation pathways to produce ATP [65]. Mixing the culture will improve mass transfer, consequently enhancing the dissolving of oxygen and boosting the Crabtree effect [66]. Besides, $\mathrm{CO}_{2}$ bubbles, produced metabolically by yeast, create an agitation environment that might favor the mass transfer effects during fermentation [64,67]. Since $\mathrm{CO}_{2}$ production is metabolically correlated with ethanol, the higher the fermentation temperature is, the higher the ethanol production, and consequently the higher the $\mathrm{CO}_{2}$ will be. Hence, at temperatures higher than $26{ }^{\circ} \mathrm{C}$, an agitation effect might be associated with $\mathrm{CO}_{2}$, and the effect of external agitation becomes negligible.

\subsubsection{Fusel Alcohol Concentration}

Fusel alcohols are 1-propanol, 2-methyl-1-propanol, and 3-methyl-1-butanol. These alcohols are mainly yielded during fermentation of sugarcane press-mud, 3-methyl-1butanol being the most representative [7]. According to Table 4, the main effects (i.e., $\mathrm{pH}$ $(\mathrm{A})$, stirring $(\mathrm{B})$, temperature $(\mathrm{C})$, and pretreatment conditions $(\mathrm{D}))$ were significant over all the tested fusel alcohols, except $\mathrm{pH}$ which did not have an impact over 1-propanol $(p<0.10)$. Moreover, the interaction between $\mathrm{pH}$ and pretreatment $(\mathrm{AD})$ influenced the production of 1-propanol, and the square of temperature $\left(C^{2}\right)$ had a significant effect over all tested alcohols $(p<0.05)$. The $\beta$ coefficients in Table 5 indicate that the main effects have a positive effect on the production of fusel alcohol. For instance, the higher the $\mathrm{pH}$ was, the higher the fusel alcohol production would be. A similar behavior of $\mathrm{pH}$ fermentation on fusel alcohol was observed by Arsahd et al. [15], who reported a significant increment $(p<0.05)$ on the fusel alcohol concentration during the fermentation of blackstrap molasses at $30^{\circ} \mathrm{C}$ under non-aerated conditions. The slight alkaline conditions of the culture medium boost the activity of the enzymes involved in the Ehrlich pathway, thus enhancing the production of fusel alcohol at higher $\mathrm{pH}$ values [68].

Table 5 shows that among the main factors, the square term of temperature $\left(\mathrm{C}^{2}\right)$ had the greatest influence on the production of fusel alcohol $(\beta=-135)$, followed by pretreatment conditions $(\beta=51.1)$, stirring $(\beta=43.5)$, and $\mathrm{pH}(\beta=35.7)$. Since $\mathrm{pH}$ did not influence the ethanol production during fermentation, as shown in Table 3, we plotted contour 
diagrams to describe the effect of $\mathrm{pH}$, temperature, and pretreatment on the concentration of main fusel alcohol. Figure 6 shows that the highest production of main fusel alcohol was found at a temperature of about $26^{\circ} \mathrm{C}$. The effect of temperature on fusel alcohol has been previously described by other authors $[16,63,69]$ and is associated with the gene expression of BAT1, which encodes for the enzymes involved in the transamination of amino acids, the first reaction in the Ehrlich pathway [12,13]. Moreover, the effect of pretreatment is clear on the fusel alcohol concentration. For instance, the production of higher alcohols was higher under hydrothermal conditions (Figure 6a-c) than under acid hydrolysis conditions (Figure 6d-f). This effect was previously reported during the fermentation of hydrolysates of sugarcane press-mud and was possibly linked to enzyme competition between the fusel aldehydes, which are intermediary in the Ehrlich metabolic pathway, and the HMF [7].
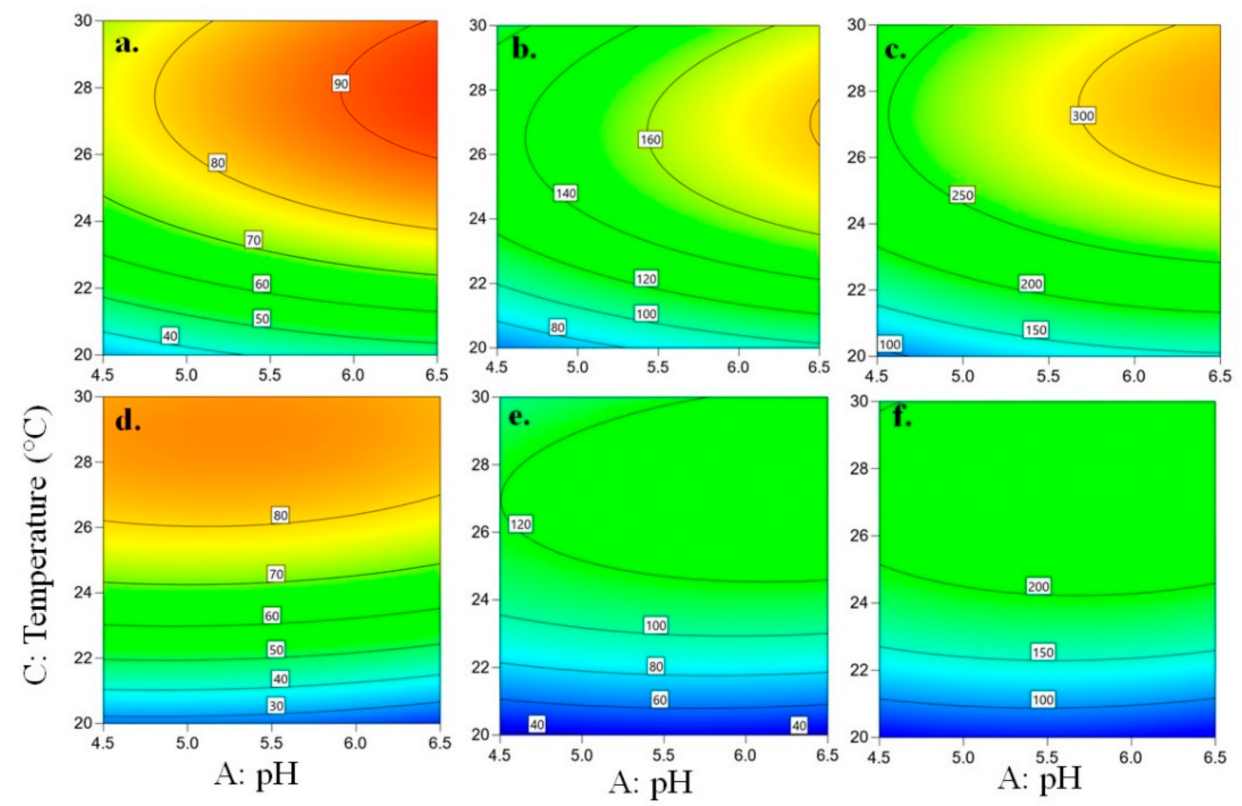

Figure 6. Effect of temperature and $\mathrm{pH}$ during the fermentation of hydrolysates produced under hydrothermal conditions to produce (a) 1-propanol (mg/L), (b) 2-methyl-1-propanol (mg/L), and (c) 3-methyl-1-butanol (mg/L), and under acid hydrolysis conditions to produce (d) 1-propanol (mg/L), (e) 2-methyl-1-propanol (mg/L), and (f) 3-methyl-1-butanol (mg/L). Stirring = $100 \mathrm{rpm}$, fermentation time $=24 \mathrm{~h}$.

\subsubsection{Condition Selection}

Based on the above description and the quadratic models, a suitable fermentation condition to produce bioethanol without affecting the ethanol concentration but reducing the amount of fusel alcohol will be $\mathrm{pH}$ : 4.5 , temperature: $30^{\circ} \mathrm{C}$, and $200 \mathrm{rpm}$. Furthermore, sugarcane press-mud must be pretreated under acid hydrolysis conditions. At these conditions, the ethanol concentration will be increased by almost $7 \%$, while reducing the fusel alcohol concentration by almost $22 \%$. In addition, the concentration of 3-methyl1-butanol, the harsher component in ESR, would be dwindled by $29 \%$. To validate this result, a second sugarcane press-mud sample was collected, pretreated, and fermented according to the selected conditions to validate the results. In the upcoming section, we present those results.

\subsection{Model Validation}

Sugarcane press-mud from Villeta was employed as feedstock to validate the model obtained from the previous experiment under laboratory conditions. Table 7 displays the initial concentration of carbohydrates on both fermentation samples, as well as the conditions of $\mathrm{pH}$, temperature, and stirring involved in each experiment. For the columns labeled Model, the predicted value for yeast cell viability is expressed in $\mathrm{Log}$ CFU/mL, 
carbohydrate conversion, and 3-methyl-1-butanol concentration in mg/L, while Villeta columns express the experimental value obtained for each parameter in comparison to the expected result.

Table 7. Fermentation results during fermentation of sugarcane press-mud collected from Villeta.

\begin{tabular}{|c|c|c|c|c|}
\hline $\begin{array}{l}\text { Fermentation } \\
\text { Conditions }\end{array}$ & \multicolumn{2}{|c|}{ Hydrothermal } & \multicolumn{2}{|c|}{ Acid Hydrolysis } \\
\hline Fermentation $\mathrm{pH}$ & \multicolumn{2}{|c|}{5.5} & \multicolumn{2}{|c|}{4.5} \\
\hline Temperature $\left({ }^{\circ} \mathrm{C}\right)$ & \multicolumn{2}{|c|}{30} & \multicolumn{2}{|c|}{30} \\
\hline Stirring (rpm) & \multicolumn{2}{|c|}{200} & \multicolumn{2}{|c|}{200} \\
\hline $\begin{array}{l}\text { Initial carbohydrate } \\
\text { concentration (GE/L) }\end{array}$ & \multicolumn{2}{|c|}{$153.78 \pm 1.04$} & \multicolumn{2}{|c|}{$142.59 \pm 52.4$} \\
\hline Fermentation Results & Model & Villeta & Model & Villeta \\
\hline $\begin{array}{l}\text { Yeast cell viability } \\
\text { (Log CFU/mL) }\end{array}$ & 2.08 & $1.89 \pm 0.05^{\mathrm{a}}$ & 1.57 & $1.74 \pm 0.06^{\mathrm{a}}$ \\
\hline $\begin{array}{l}\text { Carbohydrate } \\
\text { conversion (\%) }\end{array}$ & 95.1 & 95.6 & 99.8 & 95.6 \\
\hline Ethanol $(\mathrm{g} / \mathrm{L})$ & 86.49 & $80.06 \pm 3.75^{\mathrm{a}}$ & 92.58 & $73.88 \pm 8.32^{\mathrm{a}}$ \\
\hline $\begin{array}{l}\text { 3-methyl-1-butanol } \\
\text { (mg/L) }\end{array}$ & 296.67 & $176.02 \pm 4.20^{\mathrm{a}}$ & 210.10 & $125.94 \pm 23.98^{b}$ \\
\hline
\end{tabular}

According to Table 7, the yeast cell viability for the validation experiments were 1.89 Log CFU $/ \mathrm{mL}$ and 1.74 Log CFU $/ \mathrm{mL}$, contrasted with the anticipated $2.08 \mathrm{Log} \mathrm{CFU} / \mathrm{mL}$ and $1.57 \mathrm{Log} \mathrm{CFU} / \mathrm{mL}$ for hydrothermal and acid hydrolysis pretreatment, respectively. The error between the model and experimental results was $9.1 \%$ and $10.8 \%$ for hydrothermal and acid hydrolysis, correspondingly. Despite the difference between the model and the experimental values, a similar trend was observed. This means that when sugarcane press-mud is pretreated under acid hydrolysis conditions, the yeast cell viability is more affected than when it is pretreated under hydrothermal conditions. Concerning carbohydrate conversion, the validation experiment showed best fit to the model. Indeed, for hydrothermal and acid hydrolysis pretreatment, the experimental value was $95.6 \%$ in both cases. Besides, the error associated for each pretreatment was $0.5 \%$ and $4.2 \%$, respectively. Hence, the model was able to predict this parameter.

Ethanol concentration, as one of the most important parameters, was also assessed during validation, yet neither model was able to predict the result from Villeta's sugarcane press-mud, as the errors between the experiments and the model predictions were $20.2 \%$ and $7.4 \%$, respectively. Furthermore, ethanol concentration after acid hydrolysis decreased in $7.7 \%$, contrasted with hydrothermal pretreatment. However, according to the ANOVA test $(p<0.05)$, there was not a significant difference between both conditions when sugarcane press-mud was employed as feedstock to produce bioethanol.

On the contrary, the production of 3-methyl-1-butanol was significantly affected $(p<0.05)$, but the model also failed to predict 3-methyl-1-butanol concentration, showing errors of $40.7 \%$ and $40.0 \%$ in validation experiments for hydrothermal and acid pretreatment conditions, respectively. However, a reduction in 3-methyl-1-butanol concentration of $28.4 \%$ was observed in contrast to the expected decrease of $29.2 \%$. This result confirms that acid pretreatment does reduce the fusel alcohol concentration after fermentation as expected.

Based on the above results, it is possible to elicit that the model is not able to accurately forecast the results from biomasses obtained in different locations. However, the model will predict the expected behavior between two different conditions. Hence, the model presented in this study might be employed as a tool to select suitable conditions to reduce the amount of 3-methyl-1-butanol, which is the main fusel alcohol obtained after 
fermentation and has a detrimental effect on ESR and even on dehydration reactions to produce ethylene.

\subsection{Future Perspectives}

A biorefinery is defined as facility where organic materials from renewable sources are converted into energy, fuels, chemicals, and materials for industrial purposes [70]. Due to the complexity of organic materials, living organisms, such as fungus, bacteria, and yeasts, are commonly employed to produce the aforecited goods. However, during the conversion of organic material by using those organisms, a wide spectrum of components could be also yielded. For example, bioethanol production from fermentable sugars yield ethanol as main product, but side products such as fusel alcohol, glycerol, and acetic acid are yielded during this process [3]. Those side products have a negative impact on the upgrading of bioethanol into more valuable products such as syngas and olefins. Therefore, mitigating those side products might increase the production of syngas and olefins. This study showed that it is possible to decrease and control the formation of those side products by using different pretreatment and fermentation conditions. The novelty of this study was to determine new alternatives that could be employed within process design to increase the production of valuable goods such as syngas and ethylene that are derived from bioethanol. In addition, future studies should aim to integrate those strategies to reduce side products during the fermentation of feedstocks with high content of fermentable sugars such as sugarcane press-mud, sweet sorghum, and sugar beet, among others. The depletion of side products will increase syngas and olefins production and consequently boost the economic and social development of rural areas in Latin American countries, where biomass is an interesting alternative to decarbonize the economy.

\section{Conclusions}

A strategy to reduce the concentration of fusel alcohol during bioethanol production from sugarcane press-mud was developed by using the Box-Behnken response surface methodology. Accordingly, fermentation temperature had the greatest influence on the all the tested variables $(p<0.05)$. Moreover, the $\mathrm{pH}$ should be 4.5 because fusel alcohol can be reduced without affecting the ethanol concentration. Lastly, sugarcane press-mud should be pretreated under acid hydrolysis conditions to dwindle the concentration of fusel alcohol by almost $30 \%$ without affecting the ethanol concentration at $30{ }^{\circ} \mathrm{C}, 200 \mathrm{rpm}$, and $\mathrm{pH}=4.5$. Further studies must integrate these conditions along with the upgrading of bioethanol into $\mathrm{H}_{2}$ and light olefins to develop a suitable biorefinery concept.

Author Contributions: N.S.: conceptualization, methodology, validation, investigation, writing一original draft preparation, visualization, formal analysis. M.C.: conceptualization, resources, writing-review and editing, supervision, project administration, funding acquisition. D.R.-F.: methodology, formal analysis, writing—review and editing. M.Á.U.-L.: writing—review and editing, visualization, supervision. R.Y.R.-P.: writing-review and editing, visualization, formal analysis, supervision, conceptualization. All authors have read and agreed to the published version of the manuscript.

Funding: This research was funded by Minciencias and Universidad de La Sabana, project number ING 221 (Minciencias contract 548-2019). The doctoral scholarship granted to Nestor Sanchez was funded by Minciencias, grant number 727-2015.

Institutional Review Board Statement: Not applicable.

Informed Consent Statement: Not applicable.

Data Availability Statement: The data presented in this study are openly available in Mendeley datasets at doi:10.17632/wgv5w62yjk.1, reference number [71].

Acknowledgments: The authors are grateful to Doña Panela and Don Pedro for supplying the sugarcane press-mud samples and to Merquiand S.A who supplied the yeast strains.

Conflicts of Interest: The authors declare no conflict of interest. 


\section{References}

1. Sachs, J.D.; Schmidt-Traub, G.; Mazzucato, M.; Messner, D.; Nakicenovic, N.; Rockström, J. Six Transformations to achieve the Sustainable Development Goals. Nat. Sustain. 2019, 2, 805-814. [CrossRef]

2. Martinez-Hernandez, E.; Tibessart, A.; Campbell, G.M. Conceptual design of integrated production of arabinoxylan products using bioethanol pinch analysis. Food Bioprod. Process. 2018, 112, 1-8. [CrossRef]

3. Sanchez, N.; Ruiz, R.; Hacker, V.; Cobo, M. Impact of bioethanol impurities on steam reforming for hydrogen production: A review. Int. J. Hydrogen Energy 2020, 45, 11923-11942. [CrossRef]

4. Becerra, J.; Figueredo, M.; Cobo, M. Thermodynamic and economic assessment of the production of light olefins from bioethanol. J. Environ. Chem. Eng. 2017, 5, 1554-1564. [CrossRef]

5. Sun, J.; Wang, Y. Recent advances in catalytic conversion of ethanol to chemicals. ACS Catal. 2014, 4, 1078-1090. [CrossRef]

6. Ajit, A.; Sulaiman, A.Z.; Chisti, Y. Production of bioethanol by Zymomonas mobilis in high-gravity extractive fermentations. Food Bioprod. Process. 2017, 102, 123-135. [CrossRef]

7. Sanchez, N.; Ruiz, R.; Plazas, A.; Vasquez, J.; Cobo, M. Effect of pretreatment on the ethanol and fusel alcohol production during fermentation of sugarcane press-mud. Biochem. Eng. J. 2020, 161, 107668. [CrossRef]

8. Conesa, C.; Seguí, L.; Laguarda-Miró, N.; Fito, P. Microwaves as a pretreatment for enhancing enzymatic hydrolysis of pineapple industrial waste for bioethanol production. Food Bioprod. Process. 2016, 100, 203-213. [CrossRef]

9. Skiba, E.A.; Baibakova, O.V.; Budaeva, V.V.; Pavlov, I.N.; Vasilishin, M.S.; Makarova, E.I.; Sakovich, G.V.; Ovchinnikova, E.V.; Banzaraktsaeva, S.P.; Vernikovskaya, N.V.; et al. Pilot technology of ethanol production from oat hulls for subsequent conversion to ethylene. Chem. Eng. J. 2017, 329, 178-186. [CrossRef]

10. Sanchez, N.; Ruiz, R.Y.; Cifuentes, B.; Cobo, M. Controlling sugarcane press-mud fermentation to increase bioethanol steam reforming for hydrogen production. Waste Manag. 2019, 98, 1-13. [CrossRef]

11. Rossetti, I.; Compagnoni, M.; De Guido, G.; Pellegrini, L.A.; Ramis, G.; Dzwigaj, S. Ethylene production from diluted bioethanol solutions. Can. J. Chem. Eng. 2017, 95, 1752-1759. [CrossRef]

12. Pires, E.J.; Teixeira, J.A.; Brányik, T.; Vicente, A.A. Yeast: The soul of beer's aroma-A review of flavour-active esters and higher alcohols produced by the brewing yeast. Appl. Microbiol. Biotechnol. 2014, 98, 1937-1949. [CrossRef]

13. Hazelwood, L.A.; Daran, J.M.; van Maris, A.J.A.; Pronk, J.T.; Dickinson, J.R. The ehrlich pathway for fusel alcohol production: A century of research on Saccharomyces cerevisiae metabolism. Appl. Environ. Microbiol. 2008, 74, 2259-2266. [CrossRef] [PubMed]

14. Sanchez, N.; Ruiz, R.; Infante, N.; Cobo, M. Bioethanol Production from Cachaza as Hydrogen Feedstock: Effect of Ammonium Sulfate during Fermentation. Energies 2017, 10, 2112. [CrossRef]

15. Arshad, M.; Khan, Z.M.; Shah, F.A.; Rajoka, M.I. Optimization of process variables for minimization of byproduct formation during fermentation of blackstrap molasses to ethanol at industrial scale. Lett. Appl. Microbiol. 2008, 47, 410-414. [CrossRef] [PubMed]

16. Rollero, S.; Bloem, A.; Camarasa, C.; Sanchez, I.; Ortiz-julien, A.; Sablayrolles, J.; Dequin, S.; Mouret, J. Combined effects of nutrients and temperature on the production of fermentative aromas by Saccharomyces cerevisiae during wine fermentation. Appl. Microbiol. Biotechnol. 2015, 99, 2291-2304. [CrossRef]

17. Saerens, S.M.G.; Verbelen, P.J.; Vanbeneden, N.; Thevelein, J.M.; Delvaux, F.R. Monitoring the influence of high-gravity brewing and fermentation temperature on flavour formation by analysis of gene expression levels in brewing yeast. Appl. Microbiol. Biotechnol. 2008, 80, 1039-1051. [CrossRef] [PubMed]

18. Kłosowski, G.; Mikulski, D.; Macko, D. Influence of various yeast strains and selected starchy raw materials on production of higher alcohols during the alcoholic fermentation process. Eur. Food Res. Technol. 2015, 240, 233-242. [CrossRef]

19. Dziekońska-Kubczak, U.; Berlowska, J.; Dziugan, P.; Patelski, P.; Pielech-Przybylska, K.; Balcerek, M. Nitric Acid Pretreatment of Jerusalem Artichoke Stalks for Enzymatic Saccharification and Bioethanol Production. Energies 2018, 11, 2153. [CrossRef]

20. Mariano, A.P.B.; Unpaprom, Y.; Ramaraj, R. Hydrothermal pretreatment and acid hydrolysis of coconut pulp residue for fermentable sugar production. Food Bioprod. Process. 2020, 122, 31-40. [CrossRef]

21. Mohd Azhar, S.H.; Abdulla, R.; Jambo, S.A.; Marbawi, H.; Gansau, J.A.; Mohd Faik, A.A.; Rodrigues, K.F. Yeasts in sustainable bioethanol production: A review. Biochem. Biophys. Rep. 2017, 10, 52-61. [CrossRef]

22. Dack, R.E.; Black, G.W.; Koutsidis, G.; Usher, S.J. The effect of Maillard reaction products and yeast strain on the synthesis of key higher alcohols and esters in beer fermentations. Food Chem. 2017, 232, 595-601. [CrossRef]

23. Comelli, R.N.; Seluy, L.G.; Isla, M.A. Performance of several Saccharomyces strains for the alcoholic fermentation of sugarsweetened high-strength wastewaters: Comparative analysis and kinetic modelling. New Biotechnol. 2016, 33, 874-882. [CrossRef] [PubMed]

24. Sanchez, N.; Ruiz, R.; Rödl, A.; Cobo, M. Technical and environmental analysis on the power production from residual biomass using hydrogen as energy vector. Renew Energ 2021, 175, 825-839. [CrossRef]

25. Kliks, J.; Kawa-Rygielska, J.; Gasinski, A.; Rebas, J.; Szummy, A. Changes in the volatile composition of apple and apple/pear ciders affected by the different dilution rates in the continuous fermentation system. LWT Food Sci. Technol. 2021, 147, 111630. [CrossRef]

26. Lohrmann, J. Caracterización de Levaduras no Convencionales de la Patagonia para la Producción de Whisky con Características Sensoriales Distintivas Resumen; Trabajo de Grado Licenciatura, Licenciado en Ciencias Biológicas, Universidad Nacional del Comahue: Neuquén, Argentina, 2021. 
27. Sluiter, A.; Hames, B.; Hyman, D.; Payne, C.; Ruiz, R.; Scarlata, C.; Sluiter, J.; Templeton, D.; Nrel, J.W. Determination of Total Solids in Biomass and Total Dissolved Solids in Liquid Process Samples; National Renewable Energy Laboratory: Golden, CO, USA, 2008.

28. Pereira, F.B.; Guimarães, P.M.R.; Teixeira, J.A.; Domingues, L. Optimization of low-cost medium for very high gravity ethanol fermentations by Saccharomyces cerevisiae using statistical experimental designs. Bioresour. Technol. 2010, 101, 7856-7863. [CrossRef]

29. Walker, G.; Stewart, G. Saccharomyces cerevisiae in the Production of Fermented Beverages. Beverages 2016, 2, 30. [CrossRef]

30. Hajar, S.; Azhar, M.; Abdulla, R.; Jambo, S.A.; Marbawi, H.; Azlan, J.; Azifa, A.; Faik, M.; Francis, K. Yeasts in sustainable bioethanol production: A review. Biochem. Biophys. Rep. 2017, 10, 52-61.

31. Yang, S.; Li, Y.; Jia, D.; Yao, K.; Liu, W. The synergy of Box-Behnken designs on the optimization of polysaccharide extraction from mulberry leaves. Ind. Crop. Prod. 2017, 99, 70-78. [CrossRef]

32. Kwolek-Mirek, M.; Zadrag-Tecza, R. Comparison of methods used for assessing the viability and vitality of yeast cells. FEMS Yeast Res. 2014, 14, 1068-1079. [CrossRef] [PubMed]

33. Puligundla, P.; Smogrovicova, D.; Mok, C.; Sarathi, V.; Obulam, R. Recent developments in high gravity beer-brewing. Innov. Food Sci. Emerg. Technol. 2020, 64, 102399. [CrossRef]

34. Kawa-Rygielska, J.; Pietrzak, W.; Regiec, P.; Stencel, P. Utilization of concentrate after membrane filtration of sugar beet thin juice for ethanol production. Bioresour. Technol. 2013, 133, 134-141. [CrossRef]

35. Askarbekov, E.; Baigazieva, G.; Zhienbaeva, S.; Batyrbaeva, N.; Iztaev, A.; Uvakasova, G.; Serikbaeva, A. Comparison of different industrial strains of dry yeast for industrial fermentation of sweet sorghum syrup. Biosci. Res. 2018, 15, 1048-1062.

36. Veljković, V.B. Comment on "study of fuel properties of rubber seed oil based biodiesel" [Energy Convers. Manage. 2014; 78: 266-275] by Ahmad et al. Energy Convers. Manag. 2014, 86, 1186-1188. [CrossRef]

37. Veljković, V.B.; Veličković, A.V.; Avramović, J.M.; Stamenković, O.S. Modeling of biodiesel production: Performance comparison of Box-Behnken, face central composite and full factorial design. Chin. J. Chem. Eng. 2019, 27, 1690-1698. [CrossRef]

38. Ramaraj, R.; Unpaprom, Y. Optimization of pretreatment condition for ethanol production from Cyperus difformis by response surface methodology. 3 Biotech 2019, 9, 1-9. [CrossRef]

39. Shang, Y.H.; Zeng, Y.J.; Zhu, P.; Zhong, Q.P. Acetate metabolism of Saccharomyces cerevisiae at different temperatures during lychee wine fermentation. Biotechnol. Biotechnol. Equip. 2016, 30, 512-520. [CrossRef]

40. Whiting, G. Organic acid metabolism of yeasts during fermentation of alcoholic beverages-A review. J. Inst. Brew. 1976, 82, 84-92. [CrossRef]

41. Papapetridis, I.; Van Dijk, M.; Dobbe, A.P.A.; Metz, B.; Pronk, J.T. Improving ethanol yield in acetate-Reducing Saccharomyces cerevisiae by cofactor engineering of 6-Phosphogluconate dehydrogenase and deletion of ALD6. Microb. Cell Fact. 2016, 15, 67-83. [CrossRef] [PubMed]

42. Schober, P.; Boer, C.; Schwarte, L.A. Correlation Coefficients: Appropriate Use and Interpretation. Anesth. Analg. 2018, 126, 1763-1768. [CrossRef]

43. Wei, N.; Quarterman, J.; Kim, S.R.; Cate, J.H.D.; Jin, Y.S. Enhanced biofuel production through coupled acetic acid and xylose consumption by engineered yeast. Nat. Commun. 2013, 4, 1-8. [CrossRef]

44. Yang, X.; Wang, K.; Wang, H.; Zhang, J.; Tang, L.; Mao, Z. Control of $\mathrm{pH}$ by acetic acid and its effect on ethanol fermentation in an integrated ethanol-methane fermentation process. RSC Adv. 2016, 6, 57902-57909. [CrossRef]

45. Akillioglu, H.G.; Mogol, B.A.; Gökmen, V. Degradation of 5-hydroxymethylfurfural during yeast fermentation. Food Addit. Contam. Part A Chem. Anal. Control. Expo. Risk Assess. 2011, 28, 1629-1635. [CrossRef] [PubMed]

46. Van Maris, A.J.A.; Abbott, D.A.; Bellissimi, E.; van den Brink, J.; Kuyper, M.; Luttik, M.A.H.; Wisselink, H.W.; Scheffers, W.A.; van Dijken, J.P.; Pronk, J.T. Alcoholic fermentation of carbon sources in biomass hydrolysates by Saccharomyces cerevisiae: Current status. Antonie van Leeuwenhoek Int. J. Gen. Mol. Microbiol. 2006, 90, 391-418. [CrossRef]

47. Dziugan, P.; Balcerek, M.; Pielech-Przybylska, K.; Patelski, P. Evaluation of the fermentation of high gravity thick sugar beet juice worts for efficient bioethanol production. Biotechnol. Biofuels 2013, 6, 158. [CrossRef]

48. Lee, H.; Cho, D.H.; Kim, Y.H.; Shin, S.J.; Kim, S.B.; Han, S.O.; Lee, J.; Kim, S.W.; Park, C. Tolerance of Saccharomyces cerevisiae K35 to lignocellulose-derived inhibitory compounds. Biotechnol. Bioprocess Eng. 2011, 16, 755-760. [CrossRef]

49. Heard, G.M.; Fleet, G.H. The effects of temperature and $\mathrm{pH}$ on the growth of yeast species during the fermentation of grape juice. J. Appl. Bacteriol. 1988, 65, 23-28. [CrossRef]

50. Lu, Y.; Voon, M.K.W.; Huang, D.; Lee, P.R.; Liu, S.Q. Combined effects of fermentation temperature and pH on kinetic changes of chemical constituents of durian wine fermented with Saccharomyces cerevisiae. Appl. Microbiol. Biotechnol. 2017, 101, 3005-3014. [CrossRef]

51. Gutiérrez, A.; Chiva, R.; Sancho, M.; Beltran, G.; Arroyo-López, F.N.; Guillamon, J.M. Nitrogen requirements of commercial wine yeast strains during fermentation of a synthetic grape must. Food Microbiol. 2012, 31, 25-32. [CrossRef] [PubMed]

52. Mendieta, O.; Madrigal, G.; Castro, L.; Rodríguez, J.; Escalante, H. Sugarcane scum as a novel substrate for rapid biogas production from the non-centrifugal cane sugar agribusiness sector in developing countries. Bioresour. Technol. 2020, $297,122364$. [CrossRef] [PubMed]

53. Quiroga, E.; Moltó, J.; Conesa, J.A.; Valero, M.F.; Cobo, M. Kinetics of the catalytic thermal degradation of sugarcane residual biomass over $\mathrm{Rh}-\mathrm{Pt} / \mathrm{CeO}_{2}-\mathrm{SiO}_{2}$ for syngas production. Catalyst 2020, 10, 508. [CrossRef] 
54. Girisuta, B.; Danon, B.; Manurung, R.; Janssen, L.P.B.; Heeres, H. Experimental and kinetic modeling studies on the acid-catalysed hydrolysis of the water hyacinth plant to levulinic acid. Bioresour. Technol. 2008, 99, 8367-8375. [CrossRef] [PubMed]

55. Wang, D.; Xu, Y.; Hu, J.; Zhao, G. Fermentation Kinetics of Different Sugars by Apple Wine Yeast Saccharomyces cerevisiae. J. Inst. Brew. 2004, 110, 340-346. [CrossRef]

56. Aranda, A.; Matallana, E.; Olmo, M. Saccharomyces Yeasts I: Primary Fermentation; Elsevier: Oxford, UK, 2011 ; ISBN 9780123750211.

57. Cruz, A.L.B.; Hebly, M.; Duong, G.; Wahl, S.A.; Pronk, J.T.; Heijnen, J.J.; Daran-lapujade, P.; Gulik, W.M. Van Similar temperature dependencies of glycolytic enzymes: An evolutionary adaptation to temperature dynamics? BMC Syst. Biol. 2012, 6, 1-16. [CrossRef]

58. Kumar, R.; Kesavapillai, B. Stimulation of extracellular invertase production from spent yeast when sugarcane pressmud used as substrate through solid state fermentation. SpringerPlus 2012, 1, 1-6. [CrossRef]

59. Vilela-moura, A.; Schuller, D.; Mendes-faia, A.; Silva, R.D.; Chaves, S.R.; Sousa, M.J.; Côrte-real, M. The impact of acetate metabolism on yeast fermentative performance and wine quality: Reduction of volatile acidity of grape musts and wines. Appl. Microbiol. Biotechnol. 2011, 89, 271-280. [CrossRef]

60. Zabed, H.; Faruq, G.; Sahu, J.N.; Azirun, M.S.; Hashim, R.; Nasrulhaq Boyce, A. Bioethanol production from fermentable sugar juice. Sci. World J. 2014, 2014, 1-11. [CrossRef] [PubMed]

61. Puligundla, P.; Smogrovicova, D.; Obulam, V.S.R.; Ko, S. Very high gravity (VHG) ethanolic brewing and fermentation: A research update. J. Ind. Microbiol. Biotechnol. 2011, 38, 1133-1144. [CrossRef]

62. De Vasconcelos, J.N. Ethanol Fermentation. In Sugarcane: Agricultural Production, Bioenergy and Ethanol; Santos, F., Borem, A., Caldas, C., Eds.; Elsevier: Porto Alegre, Brazil, 2015; pp. 311-340. ISBN 9780128022399.

63. Galanakis, C.M.; Kordulis, C.; Kanellaki, M.; Koutinas, A.A.; Bekatorou, A.; Lycourghiotis, A. Effect of pressure and temperature on alcoholic fermentation by Saccharomyces cerevisiae immobilized on c-alumina pellets. Bioresour. Technol. 2012, 114, 492-498. [CrossRef] [PubMed]

64. de la Roza, C.; Laca, A.; García, L.A.; Díaz, M. Stirring and mixing effects at different cider fermentation scales. Food Bioprod. Process 2002, 80, 1-6. [CrossRef]

65. Pfeiffer, T.; Morley, A. An evolutionary perspective on the Crabtree effect. Front. Mol. Biosci. 2014, 1, 1-6. [CrossRef]

66. Mauricio, J.C.; Milla, C.; Ortega, J. Influence of oxygen on the biosynthesis of cellular fatty acids, sterols and phospholipids during alcoholic fermentation by Saccharomyces cerevisiae and Torulaspora delbrueckii. World J. Microbiol. Biotechnol. 1998, 14, 405-410. [CrossRef]

67. Rollero, S.; Roberts, S.; Bauer, F.F.; Divol, B. Agitation impacts fermentation performance as well as carbon and nitrogen metabolism in Saccharomyces cerevisiae under winemaking conditions. Aust. J. Grape Wine Res. 2015, 24, 1-8.

68. Pielech-Przybylska, K.; Balcerek, M.; Dziekońska-Kubczak, U.; Patelski, P.; Różański, M. Effect of starch liberation method and initial $\mathrm{pH}$ of sweet mashes on higher alcohols content in distillates obtained from different starchy raw materials. Process Biochem. 2018, 73, 29-37. [CrossRef]

69. Saerens, S.M.G.; Delvaux, F.; Verstrepen, K.J.; Van Dijck, P.; Thevelein, J.M.; Delvaux, F.R. Parameters Affecting Ethyl Ester Production by Saccharomyces cerevisiae during Fermentation. Appl. Environ. Microbiol. 2008, 74, 454-461. [CrossRef] [PubMed]

70. Espinoza Pérez, A.T.; Camargo, M.; Narváez Rincón, P.C.; Alfaro Marchant, M. Key challenges and requirements for sustainable and industrialized biorefinery supply chain design and management: A bibliographic analysis. Renew. Sustain. Energy Rev. 2017, 69, 350-359. [CrossRef]

71. Sanchez, N.; Rodriguez-Fontalvo, D. Dataset for fermentation of sugarcane press-mud: Effect of Temperature, Stirring, $\mathrm{pH}$, and hydrolyis on ethanol and fusel alcohol content. Mendeley Dataset 2021, V1. [CrossRef] 